This is the final peer-reviewed accepted manuscript of:

Francesc Aràndiga, Rosa Donat, Lucia Romani, Milvia Rossini: On the reconstruction of discontinuous functions using multiquadric RBF-WENO local interpolation techniques, Mathematics and Computers in Simulation, Volume 176, 2020, Pages 4-24

The final published version is available online at:

https://doi.org/10.1016/j.matcom.2020.01.018

Rights / License:

The terms and conditions for the reuse of this version of the manuscript are specified in the publishing policy. For all terms of use and more information see the publisher's website. 


\title{
On the reconstruction of discontinuous functions using multiquadric RBF-WENO local interpolation techniques
}

\author{
Francesc Aràndiga ${ }^{\mathrm{a}, *}$, Rosa Donat $^{\mathrm{a}}$, Lucia Romani $^{\mathrm{b}}$, Milvia Rossini $^{\mathrm{c}}$ \\ ${ }^{a}$ Department of Applied Mathematics, Universitat de València, Spain \\ ${ }^{b}$ Dipartimento di Matematica, Alma Mater Studiorum Università di Bologna, Piazza di Porta San Donato 5, Bologna, Italy \\ ${ }^{c}$ Dipartimento di Matematica e Applicazioni, Università di Milano-Bicocca, Via R. Cozzi 55, Milano, Italy
}

\begin{abstract}
We discuss several approaches involving the reconstruction of discontinuous one-dimensional functions using parameter-dependent multiquadric radial basis function (MQ-RBF) local interpolants combined with weighted essentially non-oscillatory (WENO) techniques, both in the computation of the locally optimized shape parameter or in the combination of RBF interpolants. We examine the accuracy of the proposed reconstruction techniques in smooth regions and their ability to avoid Gibbs phenomena close to discontinuities. In this paper, we propose a true MQ-RBF-WENO method that does not revert to the classical polynomial WENO approximation near discontinuities, as opposed to what was proposed in $[12,13]$. We present also some numerical examples that confirm the theoretical approximation orders derived in the paper.
\end{abstract}

Keywords: Local multiquadric radial basis function (RBF) interpolation; Jump discontinuity; Weighted Essentially Non-Oscillatory (WENO) interpolation; Adaptive parameter; Approximation order.

\section{Introduction and main contributions of our work}

It is a well-known fact that any global or high-order linear approximation method suffers from the Gibbs phenomenon if the function to be approximated has a jump discontinuity in the given domain. Thus, the faithful recovery of a discontinuous function is considered a challenging problem. Many different approaches for sensibly or completely reducing the Gibbs phenomenon have been suggested and studied in the literature in the last three decades (see, e.g., [4, 6, 7, 8, 9, 11, 18, 20]). However, as recently emphasized in [9], not much effort has been addressed to construct robust Radial Basis Function (RBF) approximants for functions with jump discontinuities. According to the results in [18], when the RBF approximant is obtained from multiquadric radial basis functions (MQ-RBFs) in Hardy's form $\varphi(x)=\sqrt{\delta^{2}+\|x\|_{2}^{2}}$, the Gibbs phenomenon can be considerably attenuated by fixing an initial value $\delta$ for all points and by selecting adaptively vanishing shape parameters in the vicinity of the discontinuity. In this way the MQ-RBF interpolant is locally linear and this turns out to reduce the Gibbs oscillations and to improve the accuracy of the approximation. A more effective strategy, valid for any RBF, is that of using variably scaled RBFs and to incorporate discontinuous scaling functions at or near the jump points. This strategy produces a true discontinuous interpolant and then a faithful recovery of the unknown function (see, e.g., the 1D examples in [9] and [22], and the 2D examples in [10]). However this approach requires to know or to estimate in advance the positions of the discontinuity points and an edge detection strategy is needed as preliminary step.

On the other hand, Essentially Non-Oscillatory (ENO) and Weighted ENO (WENO) polynomial techniques have become very popular since they do not require a preliminary edge detection step and allow

\footnotetext{
${ }^{*}$ Corresponding author

Email addresses: arandiga@uv.es,francesc.arandiga@uv.es (Francesc Aràndiga), rosa.m.donat@uv.es (Rosa Donat), lucia.romani@unibo.it (Lucia Romani), milvia.rossini@unimib.it (Milvia Rossini)
} 
to considerably reduce oscillations near discontinuities by the use of suitable data dependent smoothness indicators $[3,5,15,16,21,23]$.

MQ-RBF interpolants have been recently used also as reconstruction techniques in adaptive finite difference and finite volume ENO/WENO schemes for hyperbolic conservation laws $[12,13]$. In these recent papers it is numerically observed that, by locally optimizing the shape parameters of the MQ-RBF interpolant, the accuracy of the RBF-WENO scheme is somewhat enhanced in smooth regions, with respect to the polynomial WENO method based on a stencil with the same number of points. However, their proposed RBF-WENO reconstruction is not appropriate in the vicinity of a jump discontinuity, hence they need to revert to classical polynomial-based WENO reconstructions close to shocks or contact discontinuities.

Our work has been inspired by the desire to fully understand the computational results shown in these papers. Our goal is to analyze the various ways in which WENO adaptivity may be incorporated in the MQ-RBF interpolatory framework.

We show that by considering data-independent local estimates of the shape parameter, as proposed in $[12,13]$, it is possible to improve the local approximation errors in smooth regions. On the other hand, we show that these estimates lead to extremely poor approximations around jump discontinuities, which is the reason that forced the authors in $[12,13]$ to revert to WENO polynomial techniques around discontinuities in the solution. In this paper we show how WENO adaptive techniques may be incorporated in the computation of the locally optimized shape parameter in order to use the MQ-RBF-WENO reconstruction both in smooth regions and in the vicinity of jump discontinuities. We show that the proposed adaptive calculations of the shape parameter lead to accurate reconstructions and sharp profiles near jump discontinuities. A true RBFWENO interpolant, i.e., a technique for adaptively merging two MQ-RBF interpolants in such a way that the resulting interpolant can accurately approximate functions with jump discontinuities, avoiding Gibbs oscillations around jumps, is also proposed and analyzed. These new MQ-RBF-WENO techniques could be applied as effective reconstruction technique in finite difference numerical schemes for conservation laws, without having to revert to classical polynomial WENO schemes near discontinuities.

The remainder of the paper is organized as follows. In Section 2 we introduce the notation and some preliminary results on polynomial ENO/WENO interpolatory techniques. In Section 3 we review local interpolation methods based on MQ-RBFs. We show that the MQ-RBF interpolants based on 2, 3 and 4 point stencils can be conveniently approximated by a perturbation of well-known polynomial techniques. We follow this approach to determine, in Section 4, the optimal shape parameters that maximize the approximation properties of the required approximation in regions of smoothness, describing in more detail the two-point and three-point local MQ-RBF interpolation in Sections 4.1 and 4.2. In the case of two-point local MQ-RBF interpolants, we illustrate how to use WENO techniques in order to get a nonlinear estimate of the locally optimized shape parameter that allows us to achieve an approximation accuracy of order four in smooth regions and of order three near discontinuities. In Section 5 we consider a convex WENO combination of the two 3-point MQ-RBF interpolants to construct a 4-point MQ-RBF-WENO approximation similar to the ones considered in $[12,13]$. When combined with WENO-type adaptive nonlinear parameter estimation, the order of accuracy is four also in regions adjacent to a discontinuity. Moreover we propose a modified 4-point MQ-RBF-WENO approximation capable of achieving fifth-order accuracy in smooth regions. Finally, in Section 6 we present numerical evidence of the approximation order and of the faithful recovery provided by the proposed MQ-RBF-WENO reconstruction techniques in case of one-dimensional functions with jump discontinuities.

\section{Linear and nonlinear polynomial interpolation}

In this Section we set the notation used in the paper and we recall some basic results concerning local polynomial interpolation and ENO/WENO interpolatory techniques (the reader can refer to [19] and to $[15,23]$ for more details).

\subsection{Notation and basic results}

Let $b, k \in \mathbb{N}$ be such that $k \geq 2, b \geq 1$. Let $u_{j}, j=i-k+b+1, \ldots, i+b$ be $k$ values of an unknown function $u: \Omega \subset \mathbb{R} \rightarrow \mathbb{R}$ at equally spaced points $x_{j} \in \Omega, j=i-k+b+1, \ldots, i+b$, with $h=x_{j+1}-x_{j}$ 
denoting the constant grid size. In this paper, we are mainly concerned with accurate predictions to the value of the unknown function $u$ at the midpoint of the interval $\left[x_{i}, x_{i+1}\right]$, in a mesh of equally spaced points. The midpoint will be denoted as $x_{i+\frac{1}{2}}:=\left(x_{i}+x_{i+1}\right) / 2$. We shall also denote $u_{i+\frac{1}{2}}:=u\left(x_{i+\frac{1}{2}}\right)$.

Let $\mathcal{S}_{k}^{i, b}=\left\{x_{i-k+b+1}, \ldots, x_{i+b}\right\}, 1 \leq b \leq k-1$, denote any of the stencils of $k$ points containing $x_{i}$ and $x_{i+1}$. It is well-known that if $u \in C^{k}\left(\left[x_{i-k+b+1}, x_{i+b}\right]\right)$, then the unique $(k-1)$ th degree polynomial that interpolates the values of $u$ at the stencil $\mathcal{S}_{k}^{i, b}$, which we denote as $P_{k}^{i, b}(x)$, satisfies $u(x)=P_{k}^{i, b}(x)+O\left(h^{k}\right)$ for any $x \in\left[x_{i-k+b+1}, x_{i+b}\right]$. Thus,

$$
u_{i+\frac{1}{2}}=P_{k}^{i, b}\left(x_{i+\frac{1}{2}}\right)+O\left(h^{k}\right) .
$$

When evaluated at $x_{i+\frac{1}{2}}$, the polynomial approximation takes the simple form of a linear filter applied to the points of the stencil. Well known examples, to be used later on in this paper, include:

- the second-order local approximation of $u_{i+\frac{1}{2}}$ given by

$$
P_{2}^{i, 1}\left(x_{i+\frac{1}{2}}\right)=\frac{1}{2} u_{i}+\frac{1}{2} u_{i+1}
$$

- the third-order local approximations of $u_{i+\frac{1}{2}}$ given by

$$
P_{3}^{i, 1}\left(x_{i+\frac{1}{2}}\right)=-\frac{1}{8} u_{i-1}+\frac{3}{4} u_{i}+\frac{3}{8} u_{i+1}, \quad P_{3}^{i, 2}\left(x_{i+\frac{1}{2}}\right)=-\frac{1}{8} u_{i+2}+\frac{3}{4} u_{i+1}+\frac{3}{8} u_{i}
$$

- the fourth-order local approximation of $u_{i+\frac{1}{2}}$ given by

$$
P_{4}^{i, 2}\left(x_{i+\frac{1}{2}}\right)=-\frac{1}{16}\left(u_{i-1}+u_{i+2}\right)+\frac{9}{16}\left(u_{i}+u_{i+1}\right)
$$

Let $u\left[x_{i-k+b+1}, \ldots, x_{i+b}\right]$ denote the usual $(k-1)$ th order divided difference based on the values of $u$ on the stencil $\mathcal{S}_{k}^{i, b}$. It is well known that under the smoothness assumption on $u$

$$
u\left[x_{i-k+b+1}, \ldots, x_{i+b}\right]=\frac{u^{(k-1)}(\xi)}{(k-1) !}, \quad \text { for some } \quad \xi \in\left[x_{i-k+b+1}, x_{i+b}\right] .
$$

On the other hand, if $u$ is discontinuous at some point in $\left(x_{i-k+b+1}, x_{i+b}\right)$,

$$
u\left[x_{i-k+b+1}, \ldots, x_{i+b}\right]=O\left(\frac{1}{h^{k-1}}\right),
$$

(see, e.g., [15], [16] or [23]). The polynomial interpolation error at $x \in\left(x_{i-k+b+1}, x_{i+b}\right)$ depends on $h^{k}$ and on $u\left[x_{i-k+b+1}, \ldots, x_{i+b}, x\right]$, then if $u \in C^{k}\left(\left[x_{i-k+b+1}, x_{i+b}\right]\right)$ relation (1) holds. If a discontinuity occurs in the interval, $u\left[x_{i-k+b+1}, \ldots, x_{i+b}, x\right]=O\left(h^{-k}\right)$ and the interpolation error becomes $O(1)$. The loss of accuracy in the presence of discontinuities appears in the form of over/undershoots at intervals which are close to a jump discontinuity. As shown in [14] (Theorem 4.1), if the only discontinuity of $u$ belongs to a sub-interval $\left[x_{\nu}, x_{\nu+1}\right]$ of $\left[x_{i-k+b+1}, x_{i+b}\right]$, we have that $P_{k}^{i, b}(x)$ is monotone in $\left[x_{\nu}, x_{\nu+1}\right]$, but has an extremum at all other subintervals of the stencil. In practice, the over/undershoots exhibited by $P_{k}^{i, b}(x)$ are most visible at the sub-intervals near the discontinuity.

The formulas in (5), (6) indicate that the divided differences may be used as a measure of the smoothness of the function $u$ in $\left(x_{i-k+b+1}, x_{i+b}\right)$. These observations were used by Enguist, Osher, Harten and Chakravarty in $[14,15]$ in the design of the well known ENO polynomial techniques, which we briefly review for the sake of completeness and ease of reference. 


\subsection{Essentially Non-Oscillatory (ENO) local polynomial interpolation}

ENO polynomial reconstruction techniques are based on a stencil selection procedure that ensures that the interpolatory stencil remains in an area where $u$ is smooth, if possible. To construct an interpolatory polynomial of degree $k-1$ to approximate $u_{i+\frac{1}{2}}$, we may consider any of the stencils $\mathcal{S}_{k}^{i, b}$, for $1 \leq b \leq k-1$. As observed in the previous Section, divided differences can be used in the selection process. In practice, undivided differences $\left[\mathcal{S}_{k}^{i, b}\right]:=h^{k-1} u\left[x_{i-k+b+1}, \ldots, x_{i+b}\right]$ are used. Since $\left[\mathcal{S}_{k}^{i, b}\right]=O\left(h^{k-1}\right)$ if the stencil $\mathcal{S}_{k}^{i, b}$ is contained in a smooth region, and $\left[\mathcal{S}_{k}^{i, b}\right]=O(1)$ if $u$ has a jump discontinuity in $\left(x_{i-k+b+1}, x_{i+b}\right)$, by choosing $\bar{b}$ such that

$$
\left|\left[\mathcal{S}_{k}^{i, \bar{b}}\right]\right|=\min _{b=1, \ldots, k-1}\left|\left[\mathcal{S}_{k}^{i, b}\right]\right|
$$

and defining

$$
P_{k}^{E N O}(x):=P_{k}^{i, \bar{b}}(x)
$$

we ensure that

$$
u_{i+\frac{1}{2}}=P_{k}^{E N O}\left(x_{i+\frac{1}{2}}\right)+O\left(h^{k}\right),
$$

when $u$ is smooth in $\left[x_{i}, x_{i+1}\right]$, assuming that its jump discontinuities are sufficiently separated. ${ }^{1}$ Note that if $u$ has a jump discontinuity at $\left[x_{i}, x_{i+1}\right]$, the accuracy of the polynomial reconstruction is lost, but, as observed in Section 2, $P_{k}^{E N O}(x)$ remains monotone in $\left[x_{i}, x_{i+1}\right]$.

In conclusion, the ENO polynomial reconstruction maintains its full accuracy of order $k$ up to the intervals adjacent to a sufficiently isolated jump discontinuity. However, the selection process considers $k-1$ candidate stencils and then a total of $2(k-1)$ points. For $k \geq 3$, an improvement of the ENO technique, known as Weighted ENO (WENO), uses an appropriate convex combination of all possible polynomial interpolants of degree $k-1$ to provide an approximation of $u_{i+\frac{1}{2}}$ with accuracy of order $2 k-2$ in smooth regions, while maintaining the non-oscillatory properties of the ENO polynomial reconstructions.

\subsection{Weighted Essentially Non-Oscillatory (WENO) local polynomial interpolation}

The idea behind the WENO reconstruction (originally introduced by Liu et al. in the context of conservation laws [21] and improved by Jiang and Shu in [17]) is to use explicitly the information provided by the stencil containing all the $2(k-1)$ points involved in the ENO selection procedure

$$
\mathcal{S}_{2 k-2}^{i}=\left\{x_{i-k+2}, \ldots, x_{i+k-1}\right\}=\bigcup_{b=1}^{k-1} \mathcal{S}_{k}^{i, b},
$$

for constructing a convex combination of $P_{k}^{i, b}\left(x_{i+\frac{1}{2}}\right), b=1, \ldots, k-1$, that gives the interpolating polynomial $\mathcal{P}_{2 k-2}^{i}$ associated to $\mathcal{S}_{2 k-2}^{i}$ evaluated at $x_{i+\frac{1}{2}}$, so that

$$
\sum_{b=1}^{k-1} C_{k}^{b} P_{k}^{i, b}\left(x_{i+\frac{1}{2}}\right)=\mathcal{P}_{2 k-2}^{i}\left(x_{i+\frac{1}{2}}\right)=u_{i+\frac{1}{2}}+O\left(h^{2 k-2}\right),
$$

where $C_{k}^{b}, b=1, \ldots, k-1$ are suitable non-negative optimal weights satisfying the condition

$$
\sum_{b=1}^{k-1} C_{k}^{b}=1
$$

For the reader's convenience we recall that, if $k=3$, the non-negative optimal weights are $C_{3}^{1}=C_{3}^{2}=\frac{1}{2}$ and, if $k=4, C_{4}^{1}=C_{4}^{3}=\frac{3}{16}$ and $C_{4}^{2}=\frac{10}{16}$ (see, e.g., [3, 5, 21, 23] for explicit details).

\footnotetext{
${ }^{1}$ that is, there are $k$ mesh points between any two singularities, hence it is always possible to select a $k$-point stencil $\mathcal{S}_{k}^{i, b}$ so that the function is smooth in its convex hull.
} 
The (polynomial) WENO idea consists in constructing the approximation to $u_{i+\frac{1}{2}}$ by considering a suitable convex combination of $P_{k}^{i, b}\left(x_{i+\frac{1}{2}}\right), b=1, \ldots, k-1$, with coefficients that are close to the optimal weights in smooth regions and close to zero if the stencil of the corresponding interpolation polynomial is identified as non smooth. More precisely, $u_{i+\frac{1}{2}}$ is approximated by

$$
P_{2 k-2}^{W E N O}\left(x_{i+\frac{1}{2}}\right):=\sum_{b=1}^{k-1} \omega_{k}^{i, b} P_{k}^{i, b}\left(x_{i+\frac{1}{2}}\right) \quad \text { where } \quad \omega_{k}^{i, b} \geq 0, \forall b, \quad \text { and } \quad \sum_{b=1}^{k-1} \omega_{k}^{i, b}=1 .
$$

The weights $\omega_{k}^{i, b}$ are chosen to satisfy the following properties:

- $\omega_{k}^{i, b} \approx 0$ if the stencil $\mathcal{S}_{k}^{i, b}$ crosses a discontinuity so that discontinuous stencils have essentially no contribution to the convex combination in (9);

- $\omega_{k}^{i, b}$ is near the optimal weight otherwise.

In $[3,5,21,23]$, the WENO weights take the form

$$
\omega_{k}^{i, b}=\frac{\alpha_{k}^{i, b}}{\sum_{\ell=1}^{k-1} \alpha_{k}^{i, \ell}} \quad \text { with } \quad \alpha_{k}^{i, b}=\frac{C_{k}^{b}}{\left(\epsilon+I_{k}^{i, b}\right)^{\rho}}, b=1, \ldots, k-1,
$$

where

- $C_{k}^{b} \geq 0$ for all $b=1, \ldots, k-1$ and satisfy $(7),(8)$;

- $\epsilon$ is a small positive number introduced to avoid null denominator and $\rho \in \mathbb{N}$ (specifically, throughout this paper we take $\epsilon=h^{2}$ as suggested in [5] for the polynomial WENO and $\rho \geq 2$ as often done in the literature);

- $I_{k}^{i, b}, b=1, \ldots, k-1$, is a smoothness indicator for the stencil $\mathcal{S}_{k}^{i, b}$, i.e., for the function $u$ in the interval $\left[x_{i-k+b+1}, x_{i+b}\right]$. They are chosen so that if $u$ is sufficiently smooth in $\left[x_{i-k+2}, x_{i+k-1}\right]$, then $\omega_{k}^{i, b}=C_{k}^{b}+O\left(h^{k-1}\right), b=1, \ldots, k-1$, which it is sufficient to guarantee that

$$
u_{i+\frac{1}{2}}=P_{2 k-2}^{W E N O}\left(x_{i+\frac{1}{2}}\right)+O\left(h^{2 k-2}\right),
$$

as in $(7)$ (see $[5,17,21,23]$ for specific details). On the other hand, if $u$ is smooth in $\left[x_{i}, x_{i+1}\right]$ but has an isolated discontinuity at $\left(x_{i-k+2}, x_{i+k-1}\right)$, there is at least one stencil $\mathcal{S}_{k}^{i, b}$, for a specific value of $b$, that does not cross the discontinuity. Then the definition of the smoothness indicators and the weights in (10) guarantee that the approximation order is that of the interpolating ENO polynomial corresponding to that stencil, i.e.

$$
u_{i+\frac{1}{2}}=P_{2 k-2}^{W E N O}\left(x_{i+\frac{1}{2}}\right)+O\left(h^{k}\right) .
$$

If there is a discontinuity in $\left[x_{i}, x_{i+1}\right]$, the WENO construction looses all accuracy but, as in the ENO case, it does not create spurious oscillations.

\section{Local MQ-RBF interpolation}

The approximation to $u_{i+\frac{1}{2}}$ can be constructed by using a MQ-RBF interpolant of $u$ based on the $k$ points in the stencil $\mathcal{S}_{k}^{i, b}$. In this paper we take $\phi(y, \varepsilon)=\sqrt{1+y^{2} \varepsilon^{2}}$ and, as in $[12,13]$, the interpolant takes the usual form

$$
R_{k}^{i, b}(x, \varepsilon):=\sum_{j=1}^{k} \lambda_{j} \phi\left(\left|x-x_{i-k+b+j}\right|, \varepsilon\right), \quad x \in\left[x_{i-k+b+1}, x_{i+b}\right],
$$


where $\boldsymbol{\lambda}:=\left(\lambda_{1}, \ldots, \lambda_{k}\right)^{T}$ is the solution to the linear system

$$
R_{k}^{i, b}\left(x_{i+j}, \varepsilon\right)=u_{i+j}, \quad j=b+1-k, \ldots, b .
$$

Obviously, the coefficients $\lambda_{j}$ depend on the value of the shape parameter $\varepsilon$ and, when $u$ is sufficiently smooth, the value of $\varepsilon$ may be selected in order to get

$$
E_{k}^{i, b}\left(x_{i+\frac{1}{2}}, \varepsilon\right):=u_{i+\frac{1}{2}}-R_{k}^{i, b}\left(x_{i+\frac{1}{2}}, \varepsilon\right)=O\left(h^{s}\right)
$$

with $s$ as large as possible (see also $[12,13])$. Precisely, by Taylor expansions, the expression of $R_{k}^{i, b}\left(x_{i+\frac{1}{2}}, \varepsilon\right)$ can be conveniently approximated as a "perturbation" of the corresponding polynomial interpolation value, which in turn can be used to determine the optimal shape parameter.

We discuss all these issues for the cases $k=2,3,4$. We show how the local parameter $\varepsilon$ should be chosen in order to enhance the accuracy of the approximation of $u_{i+\frac{1}{2}}$ and examine the form of this approximation as a perturbed interpolation of the points in the stencil. Our discussion suggests why it is not advisable to use local MQ-RBF interpolants with $k \geq 4$.

\subsection{Local MQ-RBF 2-point interpolation}

For $\mathcal{S}_{2}^{i, 1}=\left\{x_{i}, x_{i+1}\right\}$, the local MQ-RBF interpolant of $u$ is given by

$$
R_{2}^{i, 1}(x, \varepsilon)=\lambda_{1} \phi\left(\left|x-x_{i}\right|, \varepsilon\right)+\lambda_{2} \phi\left(\left|x-x_{i+1}\right|, \varepsilon\right)
$$

with

$$
\lambda_{1}=\frac{u_{i+1} \phi(h, \varepsilon)-u_{i}}{\phi(h, \varepsilon)^{2}-1}, \quad \lambda_{2}=\frac{u_{i} \phi(h, \varepsilon)-u_{i+1}}{\phi(h, \varepsilon)^{2}-1}, \quad \phi(h, \varepsilon)=\sqrt{1+h^{2} \varepsilon^{2}} .
$$

Straightforward computations allow us to obtain the equivalent expression

$$
R_{2}^{i, 1}\left(x_{i+\frac{1}{2}}, \varepsilon\right)=\left(u_{i}+u_{i+1}\right) \frac{\sqrt{4+h^{2} \varepsilon^{2}}}{2+2 \sqrt{1+h^{2} \varepsilon^{2}}} .
$$

Expanding $\frac{\sqrt{4+h^{2} \varepsilon^{2}}}{2+2 \sqrt{1+h^{2} \varepsilon^{2}}}$ in (15) using Taylor series we get

$$
R_{2}^{i, 1}\left(x_{i+\frac{1}{2}}, \varepsilon\right)=\bar{R}_{2}^{i, 1}\left(x_{i+\frac{1}{2}}, \varepsilon\right)+O\left(h^{6}\right)
$$

with

$$
\bar{R}_{2}^{i, 1}\left(x_{i+\frac{1}{2}}, \varepsilon\right)=\left(u_{i}+u_{i+1}\right)\left(\frac{1}{2}-\frac{1}{16} h^{2} \varepsilon^{2}+\frac{11}{256} h^{4} \varepsilon^{4}\right) .
$$

Note that $\bar{R}_{2}^{i, 1}\left(x_{i+\frac{1}{2}}, \varepsilon\right)$ can be viewed as a polynomial perturbation of the second-order local approximation (2). The error function $E_{2}^{i, 1}(x, \varepsilon)=u(x)-R_{2}^{i, 1}(x, \varepsilon)$ satisfies

$$
E_{2}^{i, 1}\left(x_{i+\frac{1}{2}}, \varepsilon\right)=u_{i+\frac{1}{2}}-\bar{R}_{2}^{i, 1}\left(x_{i+\frac{1}{2}}, \varepsilon\right)+O\left(h^{6}\right)
$$

Assuming that the function $u \in C^{4}\left(\left[x_{i}, x_{i+1}\right]\right)$, and expanding $u_{i}$ and $u_{i+1}$ in (17) by Taylor expansions centered at $x_{i+\frac{1}{2}}$, we get

$$
E_{2}^{i, 1}\left(x_{i+\frac{1}{2}}, \varepsilon\right)=\frac{1}{8}\left(\varepsilon^{2} u_{i+\frac{1}{2}}-u_{i+\frac{1}{2}}^{\prime \prime}\right) h^{2}+O\left(h^{4}\right)
$$

Thus,

$$
\varepsilon_{o p t}^{2}:=\frac{u_{i+\frac{1}{2}}^{\prime \prime}}{u_{i+\frac{1}{2}}} \Rightarrow E_{2}^{i, 1}\left(x_{i+\frac{1}{2}}, \varepsilon_{o p t}\right)=O\left(h^{4}\right)
$$

i.e., it is possible to compute fourth-order local approximation to $u_{i+\frac{1}{2}}$ using the 2-point MQ-RBF-based interpolant (14) and also formula (17).

Note that this formula does not require square roots of $\varepsilon_{o p t}^{2}$, and hence avoids using complex values that may occur when $\frac{u_{i+\frac{1}{2}}^{\prime \prime}}{u_{i+\frac{1}{2}}}$ is negative. 
Remark 3.1. Note that if $u$ is smooth in $\left[x_{i}, x_{i+1}\right]$, then $u_{i+\frac{1}{2}}-\bar{R}_{2}^{i, 1}\left(x_{i+\frac{1}{2}}, 0\right)=O\left(h^{2}\right)$ since (17) reverts to (2) for $\varepsilon=0$. In fact, for any value $C$ such that $C^{2} h^{2}=O\left(h^{2}\right)$, (18) implies that $u_{i+\frac{1}{2}}-\bar{R}_{2}^{i, 1}\left(x_{i+\frac{1}{2}}, C\right)$ is at least $O\left(h^{2}\right)$.

\subsection{Local multiquadric RBF 3-point interpolation}

Following an analogous procedure, we may develop a MQ-RBF interpolant of $u$ based on the 3-point stencil $\mathcal{S}_{3}^{i, 1}=\left\{x_{i-1}, x_{i}, x_{i+1}\right\}$. Starting from the expression

$$
R_{3}^{i, 1}(x, \varepsilon)=\lambda_{1} \phi\left(\left|x-x_{i-1}\right|, \varepsilon\right)+\lambda_{2} \phi\left(\left|x-x_{i}\right|, \varepsilon\right)+\lambda_{3} \phi\left(\left|x-x_{i+1}\right|, \varepsilon\right)
$$

with

$$
\begin{aligned}
& \lambda_{1}=\frac{\left(\phi(h, \varepsilon)^{2}-1\right) u_{i-1}+\phi(h, \varepsilon)(1-\phi(2 h, \varepsilon)) u_{i}+\left(\phi(2 h, \varepsilon)-\phi(h, \varepsilon)^{2}\right) u_{i+1}}{(\phi(2 h, \varepsilon)-1)\left(-2 \phi(h, \varepsilon)^{2}+\phi(2 h, \varepsilon)+1\right)}, \\
& \lambda_{2}=\frac{-\phi(h, \varepsilon) u_{i-1}+(1+\phi(2 h, \varepsilon)) u_{i}-\phi(h, \varepsilon) u_{i+1}}{-2 \phi(h, \varepsilon)^{2}+\phi(2 h, \varepsilon)+1}, \\
& \lambda_{3}=\frac{\left(\phi(2 h, \varepsilon)-\phi(h, \varepsilon)^{2}\right) u_{i-1}+\phi(h, \varepsilon)(1-\phi(2 h, \varepsilon)) u_{i}+\left(\phi(h, \varepsilon)^{2}-1\right) u_{i+1}}{(\phi(2 h, \varepsilon)-1)\left(-2 \phi(h, \varepsilon)^{2}+\phi(2 h, \varepsilon)+1\right)}
\end{aligned}
$$

and expanding $\phi(h, \varepsilon)$ and $\phi(2 h, \varepsilon)$ by Taylor series we get

$$
R_{3}^{i, 1}\left(x_{i+\frac{1}{2}}, \varepsilon\right)=\bar{R}_{3}^{i, 1}\left(x_{i+\frac{1}{2}}, \varepsilon\right)+O\left(h^{6}\right)
$$

with

$\bar{R}_{3}^{i, 1}\left(x_{i+\frac{1}{2}}, \varepsilon\right)=\left(\frac{27}{1024} \varepsilon^{4} h^{4}-\frac{1}{8}\right) u_{i-1}+\left(\frac{171}{512} \varepsilon^{4} h^{4}-\frac{3}{16} \varepsilon^{2} h^{2}+\frac{3}{4}\right) u_{i}+\left(-\frac{441}{1024} \varepsilon^{4} h^{4}+\frac{3}{16} \varepsilon^{2} h^{2}+\frac{3}{8}\right) u_{i+1}$.

Analogously, for the 3 -point stencil $\mathcal{S}_{3}^{i, 2}=\left\{x_{i}, x_{i+1}, x_{i+2}\right\}$ we obtain

$$
R_{3}^{i, 2}\left(x_{i+\frac{1}{2}}, \varepsilon\right)=\bar{R}_{3}^{i, 2}\left(x_{i+\frac{1}{2}}\right)+O\left(h^{6}\right)
$$

with

$\bar{R}_{3}^{i, 2}\left(x_{i+\frac{1}{2}}, \varepsilon\right)=\left(\frac{27}{1024} \varepsilon^{4} h^{4}-\frac{1}{8}\right) u_{i+2}+\left(\frac{171}{512} \varepsilon^{4} h^{4}-\frac{3}{16} \varepsilon^{2} h^{2}+\frac{3}{4}\right) u_{i+1}+\left(-\frac{441}{1024} \varepsilon^{4} h^{4}+\frac{3}{16} \varepsilon^{2} h^{2}+\frac{3}{8}\right) u_{i}$.

In both cases, we can write

$$
E_{3}^{i, b}\left(x_{i+\frac{1}{2}}, \varepsilon\right)=u_{i+\frac{1}{2}}-\bar{R}_{3}^{i, b}\left(x_{i+\frac{1}{2}}, \varepsilon\right)+O\left(h^{6}\right), \quad b=1,2 .
$$

Assuming the function $u \in C^{5}\left(\left[x_{i+b-2}, x_{i+b}\right]\right)$, and expanding $\left\{u_{i+b-2}, u_{i+b-1}, u_{i+b}\right\}$ by Taylor series centered at $x_{i+\frac{1}{2}}$, we get

$$
E_{3}^{i, b}\left(x_{i+\frac{1}{2}}, \varepsilon\right)=\frac{1}{16}\left(3 u_{i+\frac{1}{2}}^{\prime} \varepsilon^{2}+u_{i+\frac{1}{2}}^{\prime \prime \prime}\right) h^{3}-\frac{3}{128}\left(3 u_{i+\frac{1}{2}} \varepsilon^{4}+u_{i+\frac{1}{2}}^{(i v)}\right) h^{4}+O\left(h^{5}\right), \quad b=1,2 .
$$

Hence

$$
\varepsilon_{o p t}^{2}:=-\frac{u_{i+\frac{1}{2}}^{\prime \prime \prime}}{3 u_{i+\frac{1}{2}}^{\prime}} \Rightarrow E_{3}^{i, b}\left(x_{i+\frac{1}{2}}, \varepsilon_{o p t}\right)=O\left(h^{4}\right), \quad b=1,2,
$$

and a fourth-order approximation can be directly computed using (21) and (22) avoiding again complex values for the shape parameter.

Remark 3.2. Note that if $u$ is smooth in $\left[x_{i-1}, x_{i+2}\right], u_{i+\frac{1}{2}}-\bar{R}_{3}^{i, b}\left(x_{i+\frac{1}{2}}, 0\right)=O\left(h^{3}\right), b=1,2$, since (21), (22) revert to (3) for $\varepsilon=0$. In fact, for any value $C$ such that $C^{2} h^{3}=O\left(h^{3}\right)$, (23) implies that $u_{i+\frac{1}{2}}-$ $\bar{R}_{3}^{i, b}\left(x_{i+\frac{1}{2}}, C\right)$ is at least $O\left(h^{3}\right)$. 


\subsection{Local multiquadric RBF 4-point interpolation}

Let us now consider the MQ-RBF interpolant of $u$ based on the 4-point stencil $\mathcal{S}_{4}^{i, 2}=\left\{x_{i-1}, x_{i}, x_{i+1}, x_{i+2}\right\}$, which is given by

$$
R_{4}^{i, 2}(x, \varepsilon)=\lambda_{1} \phi\left(\left|x-x_{i-1}\right|, \varepsilon\right)+\lambda_{2} \phi\left(\left|x-x_{i}\right|, \varepsilon\right)+\lambda_{3} \phi\left(\left|x-x_{i+1}\right|, \varepsilon\right)+\lambda_{4} \phi\left(\left|x-x_{i+2}\right|, \varepsilon\right) .
$$

For the sake of brevity, we omit the explicit expression of the interpolation coefficients and proceed as in the previous cases. Then we have

$$
R_{4}^{i, 2}\left(x_{i+\frac{1}{2}}, \varepsilon\right)=\bar{R}_{4}^{i, 2}\left(x_{i+\frac{1}{2}}, \varepsilon\right)+O\left(h^{8}\right)
$$

with

$$
\begin{aligned}
\bar{R}_{4}^{i, 2}\left(x_{i+\frac{1}{2}}\right)= & \left(-\frac{12339 \varepsilon^{6} h^{6}}{4096}+\frac{1323 \varepsilon^{4} h^{4}}{2048}-\frac{9 \varepsilon^{2} h^{2}}{64}-\frac{1}{16}\right)\left(u_{i-1}+u_{i+2}\right) \\
& +\left(\frac{9459 \varepsilon^{6} h^{6}}{4096}-\frac{1107 \varepsilon^{4} h^{4}}{2048}+\frac{9 \varepsilon^{2} h^{2}}{64}+\frac{9}{16}\right)\left(u_{i}+u_{i+1}\right) .
\end{aligned}
$$

If the function $u \in C^{6}\left(\left[x_{i-1}, x_{i+2}\right]\right)$ we get

$$
E_{4}^{i, 2}\left(x_{i+\frac{1}{2}}, \varepsilon\right)=\left(\frac{27}{128} u_{i+\frac{1}{2}} \varepsilon^{4}-\frac{36}{128} u_{i+\frac{1}{2}}^{\prime \prime} \varepsilon^{2}-\frac{3}{128} u_{i+\frac{1}{2}}^{(i v)}\right) h^{4}+O\left(h^{6}\right) .
$$

Thus, the choice

$$
\varepsilon_{o p t}^{2}:=\frac{2 u_{i+\frac{1}{2}}^{\prime \prime} \pm \sqrt{4\left(u_{i+\frac{1}{2}}^{\prime \prime}\right)^{2}+u_{i+\frac{1}{2}} u_{i+\frac{1}{2}}^{(i v)}}}{3 u_{i+\frac{1}{2}}}
$$

guarantees

$$
E_{4}^{i, 2}\left(x_{i+\frac{1}{2}}, \varepsilon_{\text {opt }}\right)=O\left(h^{6}\right) .
$$

Remark 3.3. There is an obvious difference with respect to the previous cases: the square root in (28) may lead to complex values in the computation of the optimal shape parameter. Hence, local MQ-RBF interpolations based on more than 3 points are of marginal interest from a practical point of view and, thus, will not be investigated hereafter.

\section{Estimation of the optimal shape parameter and accuracy}

In practice, the value of $\varepsilon_{\mathrm{opt}}^{2}$ needs to be approximated, since $u$ is unknown. Let $\varepsilon^{2}$ be an approximation of $\varepsilon_{\text {opt }}^{2}$ such that

$$
\varepsilon^{2}=\varepsilon_{o p t}^{2}+O\left(h^{p}\right) .
$$

Then, from (18), (23) and (27), we easily obtain

$$
\begin{aligned}
& E_{2}^{i, 1}\left(x_{i+\frac{1}{2}}, \varepsilon\right)=O\left(h^{\min \{p+2,4\}}\right), \\
& E_{3}^{i, b}\left(x_{i+\frac{1}{2}}, \varepsilon\right)=O\left(h^{\min \{p+3,4\}}\right), \quad b=1,2, \\
& E_{4}^{i, 2}\left(x_{i+\frac{1}{2}}, \varepsilon\right)=O\left(h^{\min \{p+4,6\}}\right) .
\end{aligned}
$$

Consequently, in order to preserve the order of the approximation error obtained for the optimal value of the shape parameter, it is sufficient to approximate $\varepsilon_{o p t}^{2}$

- to second-order accuracy, when using local MQ-RBF interpolation based on two or four points, and

- to first-order accuracy, when using local MQ-RBF interpolation based on three points.

Remark 4.1. We remark that formulas (17), (21), (22) and (26), revert to (2), (3) and (4) when $\varepsilon=0$.

In practice, the approximations to the optimal shape parameters will be computed using available values of the function $u$. In the next Sections we examine and compare different alternatives. The proofs of the results can be easily obtained by Taylor expansions and are omitted for the sake of conciseness. 


\subsection{Local $M Q-R B F$ 2-point interpolation}

When $k=2$, we may obtain a second-order approximation of $\varepsilon_{\mathrm{opt}}^{2}=\frac{u_{i+\frac{1}{2}}^{\prime \prime}}{u_{i+\frac{1}{2}}}$ using $u_{i-1}, u_{i+2}$ as additional points.

Proposition 4.2. Let $u \in C^{4}\left(\left[x_{i-1}, x_{i+2}\right]\right)$, then

$$
\bar{u}_{i+\frac{1}{2}}^{\prime \prime}:=\frac{u_{i-1}-u_{i}-u_{i+1}+u_{i+2}}{2 h^{2}}=u_{i+\frac{1}{2}}^{\prime \prime}+O\left(h^{2}\right), \quad \bar{u}_{i+\frac{1}{2}}:=\frac{u_{i}+u_{i+1}}{2}=u_{i+\frac{1}{2}}+O\left(h^{2}\right),
$$

hence

$$
\varepsilon_{\text {lin }}^{2}:=\frac{\bar{u}_{i+\frac{1}{2}}^{\prime \prime}}{\bar{u}_{i+\frac{1}{2}}}=\varepsilon_{o p t}^{2}+O\left(h^{2}\right),
$$

and thus, in light of (30),

$$
E_{2}^{i, 1}\left(x_{i+\frac{1}{2}}, \varepsilon_{l i n}\right)=O\left(h^{4}\right) .
$$

The next Proposition analyzes the accuracy when the function is smooth in $\left[x_{i}, x_{i+1}\right]$ but has a discontinuity in one of its adjacent intervals, so that the set of points used to compute the linear approximation to $u_{i+\frac{1}{2}}^{\prime \prime}$ in (32) crosses the discontinuity.

Proposition 4.3. If $u$ has a discontinuity at $\left[x_{i-1}, x_{i}\right]$ (or $\left[x_{i+1}, x_{i+2}\right]$ ) then $\varepsilon_{\text {lin }}^{2}$ in (32) satisfies

$$
\varepsilon_{l i n}^{2}=O\left(\frac{1}{h^{2}}\right)
$$

and so, in light of (18)

$$
E_{2}^{i, 1}\left(x_{i+\frac{1}{2}}, \varepsilon_{l i n}\right)=O(1)
$$

According to Remark 3.1, if $u$ is smooth in $\left[x_{i}, x_{i+1}\right], E_{2}^{i, 1}\left(x_{i+\frac{1}{2}}, \varepsilon\right)$ is at least $O\left(h^{2}\right)$ for any constant (independent of $h$ ) value of $\varepsilon$. This suggests to consider a simple alternative computation of the shape parameter defined in (32) that at least preserves a second-order accuracy, even at intervals close to a jump discontinuity.

Proposition 4.4. Assume that $\alpha=\max _{x \in \Omega}\left|\frac{u^{\prime \prime}(x)}{u(x)}\right|<+\infty$, and define

$$
\varepsilon_{\text {alt }}^{2}:=\operatorname{sign}\left(\varepsilon_{\text {lin }}^{2}\right) \min \left(a b s\left(\varepsilon_{\text {lin }}^{2}\right), c \alpha\right), \quad c>1 .
$$

Then:

1. If $u$ is smooth in $\left[x_{i-1}, x_{i+2}\right], \varepsilon_{\text {alt }}^{2}=\varepsilon_{\text {lin }}^{2}$ and $E_{2}^{i, 1}\left(x_{i+\frac{1}{2}}, \varepsilon_{\text {alt }}\right)=O\left(h^{4}\right)$.

2. If $u$ has a discontinuity in $\left[x_{i-1}, x_{i}\right]$ (or $\left.\left[x_{i+1}, x_{i+2}\right]\right), E_{2}^{i, 1}\left(x_{i+\frac{1}{2}}, \varepsilon_{a l t}\right)=O\left(h^{2}\right)$.

We shall see that $\varepsilon_{\text {alt }}$ provides a simple, and yet effective, selection of the shape parameter that keeps the optimal accuracy expected in smooth regions while maintaining a second-order accurate approximation at intervals adjacent to a jump discontinuity.

A more effective estimation of the optimal shape parameter $\varepsilon_{\text {opt }}$ can be obtained via adaptive non linear WENO-like strategies that provide an accurate local approximation of $u_{i+\frac{1}{2}}$ up to the interval that contains the discontinuity. The starting point is the following observation. Let $u \in C^{4}\left(\left[x_{i-1}, x_{i+2}\right]\right)$, and define

$$
u_{i+\frac{1}{2}, L}^{\prime \prime}:=\frac{u_{i-1}-2 u_{i}+u_{i+1}}{h^{2}}, \quad u_{i+\frac{1}{2}, R}^{\prime \prime}:=\frac{u_{i}-2 u_{i+1}+u_{i+2}}{h^{2}},
$$

then

$$
\frac{1}{2} u_{i+\frac{1}{2}, L}^{\prime \prime}+\frac{1}{2} u_{i+\frac{1}{2}, R}^{\prime \prime}=\bar{u}_{i+\frac{1}{2}}^{\prime \prime}
$$

with $\bar{u}_{i+\frac{1}{2}}^{\prime \prime}$ defined in (31). Note that $u_{i+\frac{1}{2}, L, R}^{\prime \prime}=u_{i+\frac{1}{2}}^{\prime \prime}+O(h)$.

The following Lemma examines the accuracy obtained when we substitute the optimal coefficients 1/2, $1 / 2$ by the corresponding WENO weights (10). 
Lemma 4.5. Let

$$
\omega_{3}^{i, b}=\frac{\alpha_{3}^{i, b}}{\sum_{\ell=1}^{2} \alpha_{3}^{i, \ell}}, \quad \alpha_{3}^{i, b}=\frac{1 / 2}{\left(h^{2}+I_{3}^{i, b}\right)^{2}}, \quad b=1,2,
$$

where the smoothness indicators $I_{3}^{i, b}, b=1,2$ are defined as in [17] by

$$
\begin{aligned}
I_{3}^{i, 1} & =\frac{13}{12}\left(u_{i-1}-2 u_{i}+u_{i+1}\right)^{2}+\frac{1}{4}\left(u_{i-1}-4 u_{i}+3 u_{i+1}\right)^{2} \\
I_{3}^{i, 2} & =\frac{13}{12}\left(u_{i}-2 u_{i+1}+u_{i+2}\right)^{2}+\frac{1}{4}\left(u_{i+2}-u_{i}\right)^{2}
\end{aligned}
$$

Then:

1. If $u \in C^{4}\left(\left[x_{i-1}, x_{i+2}\right]\right), \omega_{3}^{i, b}=\frac{1}{2}+O\left(h^{2}\right), b=1,2$ and,

$$
\omega_{3}^{i, 1} u_{i+\frac{1}{2}, L}^{\prime \prime}+\omega_{3}^{i, 2} u_{i+\frac{1}{2}, R}^{\prime \prime}=u_{i+\frac{1}{2}}^{\prime \prime}+O\left(h^{2}\right) .
$$

2. If $u$ has a discontinuity at $\left[x_{i+1}, x_{i+2}\right]$ (or $\left.\left[x_{i-1}, x_{i}\right]\right)$ and $u$ is at least $C^{3}$ in $\left[x_{i-1}, x_{i+1}\right]$ (or in $\left.\left[x_{i}, x_{i+2}\right]\right)$, then $\omega_{3}^{i, 1}=1+O\left(h^{4}\right), \omega_{3}^{i, 2}=O\left(h^{4}\right)\left(\right.$ or $\left.\omega_{3}^{i, 1}=O\left(h^{4}\right), \omega_{3}^{i, 2}=1+O\left(h^{4}\right)\right)$ and

$$
\omega_{3}^{i, 1} u_{i+\frac{1}{2}, L}^{\prime \prime}+\omega_{3}^{i, 2} u_{i+\frac{1}{2}, R}^{\prime \prime}=u_{i+\frac{1}{2}}^{\prime \prime}+O(h)
$$

Then the next result follows easily.

Proposition 4.6. Define

$$
\varepsilon_{w e n}^{2}:=\frac{\omega_{3}^{i, 1} u_{i+\frac{1}{2}, L}^{\prime \prime}+\omega_{3}^{i, 2} u_{i+\frac{1}{2}, R}^{\prime \prime}}{\left(u_{i}+u_{i+1}\right) / 2} .
$$

Then:

1. If $u \in C^{4}\left(\left[x_{i-1}, x_{i+2}\right]\right)$,

$$
\varepsilon_{w e n}^{2}=\varepsilon_{o p t}^{2}+O\left(h^{2}\right) \quad \Rightarrow \quad E_{2}^{i, 1}\left(x_{i+\frac{1}{2}}, \varepsilon_{w e n}\right)=O\left(h^{4}\right)
$$

2. If $u$ has a discontinuity at $\left[x_{i+1}, x_{i+2}\right]$ (or $\left.\left[x_{i-1}, x_{i}\right]\right)$ and $u$ is at least $C^{3}$ in $\left[x_{i-1}, x_{i+1}\right]$ (or in $\left.\left[x_{i}, x_{i+2}\right]\right)$,

$$
\varepsilon_{w e n}^{2}=\varepsilon_{o p t}^{2}+O(h) \Rightarrow E_{2}^{i, 1}\left(x_{i+\frac{1}{2}}, \varepsilon_{w e n}\right)=O\left(h^{3}\right) .
$$

Lemma 4.5 is exploited further in Section 4.2 to derive a true WENO-RBF local interpolant, by using the above nonlinear weights to merge the two perturbed polynomial approximations derived in Section 3.2 .

Remark 4.7. We remark that the 2-point $M Q-R B F$ reconstruction may provide an accurate approximation to $u_{i+\frac{1}{2}}$ right up to the interval that contains a jump discontinuity by choosing the shape parameter WENO adaptively as in (36). In this case, the computation of the shape parameter requires the values of $u$ on the stencil $\left\{x_{i-1}, x_{i}, x_{i+1}, x_{i+2}\right\}$. The approximation order of the corresponding MQ-RBF-WENO interpolant in smooth regions is 4 , and 3 next to a jump discontinuity, just as the corresponding WENO polynomial reconstruction based on the same points.

\subsection{Local $M Q-R B F$ 3-point interpolation}

In Section 3.2 we have shown that $\varepsilon_{\mathrm{opt}}^{2}:=-\frac{u_{i+\frac{1}{2}}^{\prime \prime \prime}}{3 u_{i+\frac{1}{2}}^{\prime}}$ provides $E_{3}^{i, b}\left(x_{i+\frac{1}{2}}, \varepsilon_{\text {opt }}\right)=O\left(h^{4}\right), b=1,2$. In light of (29) and (30) and with the same arguments of Section 4.1, we may easily construct data dependent approximations of $\varepsilon_{\mathrm{opt}}^{2}$ leading to a fourth-order approximation of $u_{i+\frac{1}{2}}$ in smooth regions. 
Proposition 4.8. Let $u \in C^{5}\left(\left[x_{i-1}, x_{i+2}\right]\right)$, then

$$
\bar{u}_{i+\frac{1}{2}}^{\prime \prime \prime}:=\frac{-u_{i-1}+3 u_{i}-3 u_{i+1}+u_{i+2}}{h^{3}}=u_{i+\frac{1}{2}}^{\prime \prime \prime}+O\left(h^{2}\right) \quad \text { and } \quad \bar{u}_{i+\frac{1}{2}}^{\prime}:=\frac{u_{i+1}-u_{i}}{h}=u_{i+\frac{1}{2}}^{\prime}+O\left(h^{2}\right) .
$$

Hence

$$
\varepsilon_{\text {lin }}^{2}:=-\frac{\bar{u}_{i+\frac{1}{2}}^{\prime \prime \prime}}{3 \bar{u}_{i+\frac{1}{2}}^{\prime}}=\varepsilon_{o p t}^{2}+O\left(h^{2}\right),
$$

and in light of (30)

$$
E_{3}^{i, b}\left(x_{i+\frac{1}{2}}, \varepsilon_{\text {lin }}\right)=O\left(h^{4}\right) .
$$

Note that $\varepsilon_{\text {lin }}^{2}$ in (38) involves the points $\left\{x_{i-1}, x_{i}, x_{i+1}, x_{i+2}\right\}$. When the function is smooth in $\left[x_{i-1}, x_{i+1}\right]$ but has a discontinuity at $\left[x_{i+1}, x_{i+2}\right]$, the accuracy of $\bar{R}_{3}^{i, 1}\left(x_{i+\frac{1}{2}}, \varepsilon_{l i n}^{2}\right)$ is lost, as stated in the next Proposition.

Proposition 4.9. If $u$ has a discontinuity in $\left[x_{i-1}, x_{i}\right]$ (or in $\left[x_{i+1}, x_{i+2}\right]$ ) then $\varepsilon_{\text {lin }}^{2}$ in (38) satisfies

$$
\varepsilon_{l i n}^{2}=O\left(\frac{1}{h^{3}}\right),
$$

hence

$$
E_{3}^{i, b}\left(x_{i+\frac{1}{2}}, \varepsilon_{\text {lin }}\right)=O\left(\frac{1}{h^{2}}\right) \quad \text { for } b=1 \quad(\text { or } b=2) .
$$

Proof. In light of (23) and observing that $\varepsilon_{\text {lin }}^{4}=O\left(\frac{1}{h^{6}}\right)$, the claimed result is obtained.

According to Remark 3.2, if $u$ is smooth in $\left[x_{i-2+b}, x_{i+b}\right], b=1,2, E_{3}^{i, b}\left(x_{i+\frac{1}{2}}, \varepsilon\right)$ is at least $O\left(h^{3}\right)$ for any value of $\varepsilon$ independent of $h$. Hence, as in the two-point case, we may provide an estimate of the shape parameter that maintains at least third-order accuracy, except when the singularity belongs to $\left[x_{i}, x_{i+1}\right]$.

Proposition 4.10. Assume that $\alpha=\max _{x \in \Omega}\left|-\frac{u^{\prime \prime \prime}(x)}{3 u^{\prime}(x)}\right|<+\infty$, and define

$$
\varepsilon_{\text {alt }}^{2}=\operatorname{sign}\left(\varepsilon_{\text {lin }}^{2}\right) \min \left(a b s\left(\varepsilon_{\text {lin }}^{2}\right), c \alpha\right), \quad c>1 .
$$

Then:

1. If $u \in C^{5}\left(\left[x_{i-1}, x_{i+2}\right]\right)$,

$$
\varepsilon_{\text {alt }}=\varepsilon_{\text {lin }}, \quad \Rightarrow \quad E_{3}^{i, b}\left(x_{i+\frac{1}{2}}, \varepsilon_{\text {alt }}\right)=O\left(h^{4}\right), \quad b=1,2 .
$$

2. If $u$ has a discontinuity in $\left[x_{i-1}, x_{i}\right]$ (or in $\left[x_{i+1}, x_{i+2}\right]$ ),

$$
\varepsilon_{\text {alt }}=\varepsilon_{\text {opt }}+O(1) \quad \Rightarrow \quad E_{3}^{i, 2}\left(x_{i+\frac{1}{2}}, \varepsilon_{\text {alt }}\right)=O\left(h^{3}\right),\left(\text { or } E_{3}^{i, 1}\left(x_{i+\frac{1}{2}}, \varepsilon_{\text {alt }}\right)=O\left(h^{3}\right)\right) .
$$

It is interesting to note that a WENO-like computation of the shape parameter like the one carried out in Section 4.1, does not really make sense in this case. Nevertheless, further improvements can be obtained by considering a full WENO approach to merge the two 3-point MQ-RBF interpolants.

\section{4-point local MQ-RBF-WENO interpolants}

Let us consider the expressions $\bar{R}_{3}^{i, 1}\left(x_{i+\frac{1}{2}}, \varepsilon\right)$ and $\bar{R}_{3}^{i, 2}\left(x_{i+\frac{1}{2}}, \varepsilon\right)$ in (21) and (22), derived in Section 3.2 by considering the MQ-RBF interpolation based on the stencils $\mathcal{S}_{3}^{i, b}, b=1,2$, and their convex combination

$$
R_{4}^{W E N O}\left(x_{i+\frac{1}{2}}\right):=\sum_{b=1}^{2} \omega_{3}^{i, b} \bar{R}_{3}^{i, b}\left(x_{i+\frac{1}{2}}\right)
$$


with $\omega_{3}^{i, b}$ the weights defined in Lemma 4.5. Thus,

$$
\begin{aligned}
R_{4}^{W E N O}\left(x_{i+\frac{1}{2}}\right) & =\omega_{3}^{i, 1}\left(\frac{27}{1024} \varepsilon^{4} h^{4}-\frac{1}{8}\right) u_{i-1} \\
& +\left[\omega_{3}^{i, 1}\left(\frac{171}{512} \varepsilon^{4} h^{4}-\frac{3}{16} \varepsilon^{2} h^{2}+\frac{3}{4}\right)+\omega_{3}^{i, 2}\left(-\frac{441}{1024} \varepsilon^{4} h^{4}+\frac{3}{16} \varepsilon^{2} h^{2}+\frac{3}{8}\right)\right] u_{i} \\
& +\left[\omega_{3}^{i, 1}\left(-\frac{441}{1024} \varepsilon^{4} h^{4}+\frac{3}{16} \varepsilon^{2} h^{2}+\frac{3}{8}\right)+\omega_{3}^{i, 2}\left(\frac{171}{512} \varepsilon^{4} h^{4}-\frac{3}{16} \varepsilon^{2} h^{2}+\frac{3}{4}\right)\right] u_{i+1} \\
& +\omega_{3}^{i, 2}\left(\frac{27}{1024} \varepsilon^{4} h^{4}-\frac{1}{8}\right) u_{i+2} .
\end{aligned}
$$

Assuming the function $u \in C^{5}\left(\left[x_{i-1}, x_{i+2}\right]\right)$, and evaluating $u_{i-1}, u_{i}, u_{i+1}$ and $u_{i+2}$ by Taylor expansions centered at $x_{i+\frac{1}{2}}$, we easily obtain the formulation

$$
\begin{aligned}
R_{4}^{W E N O}\left(x_{i+\frac{1}{2}}\right) & =\left(\omega_{3}^{i, 1}+\omega_{3}^{i, 2}\right) u_{i+\frac{1}{2}}-\frac{1}{16}\left(3 u_{i+\frac{1}{2}}^{\prime} \varepsilon^{2}+u_{i+\frac{1}{2}}^{\prime \prime \prime}\right)\left(\omega_{3}^{i, 1}-\omega_{3}^{i, 2}\right) h^{3} \\
& -\frac{3}{128}\left(3 u_{i+\frac{1}{2}} \varepsilon^{4}+u_{i+\frac{1}{2}}^{(i v)}\right)\left(\omega_{3}^{i, 1}+\omega_{3}^{i, 2}\right) h^{4}+O\left(h^{5}\right),
\end{aligned}
$$

that allows us to easily get the following result.

Proposition 5.1. 1. If $u \in C^{5}\left(\left[x_{i-1}, x_{i+2}\right]\right)$ then

$$
u_{i+\frac{1}{2}}-R_{4}^{W E N O}\left(x_{i+\frac{1}{2}}, \varepsilon\right)=\frac{3}{128}\left(u_{i+\frac{1}{2}}^{(i v)}+3 \varepsilon^{4} u_{i+\frac{1}{2}}\right) h^{4}+O\left(h^{5}\right) .
$$

2. If $u \in C^{5}\left(\left[x_{i-1}, x_{i+1}\right]\right)$ and has a jump discontinuity at $\left[x_{i+1}, x_{i+2}\right]$ (or if $u \in C^{5}\left(\left[x_{i}, x_{i+2}\right]\right.$ ) and has a jump discontinuity at $\left.\left[x_{i-1}, x_{i}\right]\right)$ then

$$
u_{i+\frac{1}{2}}-R_{4}^{W E N O}\left(x_{i+\frac{1}{2}}, \varepsilon\right)=\frac{1}{16}\left(3 u_{i+\frac{1}{2}}^{\prime} \varepsilon^{2}+u_{i+\frac{1}{2}}^{\prime \prime \prime}\right) h^{3}-\frac{3}{128}\left(3 u_{i+\frac{1}{2}} \varepsilon^{4}+u_{i+\frac{1}{2}}^{(i v)}\right) h^{4}+O\left(h^{4}\right) .
$$

Proof. By the properties of the WENO weights in Lemma 4.5, relation (43) follows directly from (42) and (44) from (23).

As expected, the weights will tend to select the 3-point MQ-RBF interpolant based on the stencil that does not cross the discontinuity and the leading order of the error in the convex combination corresponds to that of the selected 3-point MQ-RBF interpolant. According to (24) and to the results of Section 4.2, we know that an improvement of the order of accuracy is possible whenever we provide a good estimation of the optimal parameter $\varepsilon_{\mathrm{opt}}^{2}=-\frac{u_{i+\frac{1}{2}}^{\prime \prime \prime}}{3 u_{i+\frac{1}{2}}^{\prime}}$. Indeed the two stencils used for constructing the MQ-RBF-WENO approximation (40) can be used to provide WENO-type adaptive nonlinear estimation of $\varepsilon_{\mathrm{opt}}^{2}$. The possible optimal parameter estimations will be discussed in Section 5.2 while in Section 5.1 we propose a modified 4-point MQ-RBF-WENO technique that further enhances the accuracy in smooth regions by annihilating the first term in (43).

\subsection{Enhancing accuracy: a modified 4-point $M Q-R B F-W E N O$}

The choice

$$
\varepsilon_{o p t}^{4}:=-\frac{u_{i+\frac{1}{2}}^{(i v)}}{3 u_{i+\frac{1}{2}}},
$$

would obviously annihilate the first term in (43), but in order to use it in (41) we would also need the value of $\varepsilon_{o p t}^{2}$, which could be complex. Note that the $\varepsilon^{2} h^{2}$ term in (41) is of the form

$$
\frac{3}{16}\left(u_{i+1}-u_{i}\right)\left(\omega_{3}^{i, 1}-\omega_{3}^{i, 2}\right) \varepsilon^{2} h^{2} .
$$


Taking into account that in smooth regions $\omega_{3}^{i, 1}=\omega_{3}^{i, 2}=\frac{1}{2}+O\left(h^{2}\right)$, it turns out that (45) is of order $h^{5}$ and its contribution to the convex combination can be neglected as it is of the order of the error. This observation leads us to consider the following modified 4-point $M Q-R B F-W E N O$ reconstruction technique:

$$
G_{4}^{W E N O}\left(x_{i+\frac{1}{2}}, \varepsilon\right):=\sum_{b=1}^{2} \omega_{3}^{i, b} G_{3}^{i, b}\left(x_{i+\frac{1}{2}}, \varepsilon\right)
$$

where

$$
\begin{aligned}
& G_{3}^{i, 1}\left(x_{i+\frac{1}{2}}, \varepsilon\right)=\left(\frac{27}{1024} \varepsilon^{4} h^{4}-\frac{1}{8}\right) u_{i-1}+\left(\frac{171}{512} \varepsilon^{4} h^{4}+\frac{3}{4}\right) u_{i}+\left(-\frac{441}{1024} \varepsilon^{4} h^{4}+\frac{3}{8}\right) u_{i+1}, \\
& G_{3}^{i, 2}\left(x_{i+\frac{1}{2}}, \varepsilon\right)=\left(\frac{27}{1024} \varepsilon^{4} h^{4}-\frac{1}{8}\right) u_{i+2}+\left(\frac{171}{512} \varepsilon^{4} h^{4}+\frac{3}{4}\right) u_{i+1}+\left(-\frac{441}{1024} \varepsilon^{4} h^{4}+\frac{3}{8}\right) u_{i} .
\end{aligned}
$$

In regions of smoothness, it has the capability to provide fifth-order approximations to $u_{i+\frac{1}{2}}$. In practice, we need to estimate $\varepsilon_{\text {opt }}$ using available values of $u$. The obvious choice is to consider the following centered construction:

$$
\bar{u}_{i+\frac{1}{2}}^{(i v)}:=\frac{1}{6 h^{4}}\left(u_{i-2}-3 u_{i-1}+2 u_{i}+2 u_{i+1}-3 u_{i+2}+u_{i+3}\right) .
$$

The results are summarized in the following Proposition.

Proposition 5.2. Let

$$
\varepsilon_{l i n}^{4}:=-\frac{u_{i-2}-3 u_{i-1}+2 u_{i}+2 u_{i+1}-3 u_{i+2}+u_{i+3}}{3 h^{4}\left(u_{i}+u_{i+1}\right)} .
$$

Then:

1. If $u$ is smooth in $\left[x_{i-2}, x_{i+3}\right]$ then $\varepsilon_{\text {lin }}^{4}=\varepsilon_{o p t}^{4}+O\left(h^{2}\right)$ and

$$
u_{i+\frac{1}{2}}-G_{4}^{W E N O}\left(x_{i+\frac{1}{2}}, \varepsilon_{l i n}\right)=O\left(h^{5}\right) .
$$

2. If $u$ has a discontinuity in $\left[x_{i-2}, x_{i}\right]$ (or $\left.\left[x_{i+1}, x_{i+3}\right]\right)$ then $\varepsilon_{\text {lin }}^{4}=O\left(h^{-4}\right)$ and

$$
u_{i+\frac{1}{2}}-G_{4}^{W E N O}\left(x_{i+\frac{1}{2}}, \varepsilon_{l i n}\right)=O(1) .
$$

Proof. If $u$ is sufficiently smooth in $\left[x_{i-2}, x_{i+3}\right]$, then $\bar{u}_{i+\frac{1}{2}}^{(i v)}$ in (47) satisfies $\bar{u}_{i+\frac{1}{2}}^{(i v)}=u_{i+\frac{1}{2}}^{(i v)}+O\left(h^{2}\right)$. Hence $\varepsilon_{\text {lin }}^{4}=\varepsilon_{\text {opt }}^{4}+O\left(h^{2}\right)$. In addition, under these smoothness assumptions

$$
u_{i+\frac{1}{2}}-G_{4}^{W E N O}\left(x_{i+\frac{1}{2}}, \varepsilon\right)=\frac{3}{128}\left(u_{i+\frac{1}{2}}^{(i v)}+3 \varepsilon^{4} u_{i+\frac{1}{2}}\right) h^{4}+O\left(h^{5}\right)
$$

from which we obtain the first part of the proposition.

On the other hand if $u$ has a discontinuity in $\left[x_{i-2}, x_{i}\right]$ (or $\left.\left[x_{i+1}, x_{i+3}\right]\right)$ and it is smooth in $\left[x_{i}, x_{i+3}\right]$ (smooth in $\left.\left[x_{i-1}, x_{i}\right]\right)$ then $\bar{u}_{i+\frac{1}{2}}^{(i v)}$ in $(47)$ satisfies $\bar{u}_{i+\frac{1}{2}}^{(i v)}=O\left(h^{-4}\right)$, hence $\varepsilon_{l i n}^{4}=O\left(h^{-4}\right)$. In this case, it is easy to deduce that

$$
u_{i+\frac{1}{2}}-G_{4}^{W E N O}\left(x_{i+\frac{1}{2}}, \varepsilon\right)=\frac{1}{16}\left(u_{i+\frac{1}{2}}^{\prime \prime \prime}\right) h^{3}-\frac{3}{128}\left(3 u_{i+\frac{1}{2}} \varepsilon^{4}+u_{i+\frac{1}{2}}^{(i v)}\right) h^{4}+O\left(h^{4}\right),
$$

from which we get the second result stated in the proposition.

As in previous Sections, we may easily consider simpler alternatives to define the shape parameter in order to maintain at least third order accuracy in the approximation to $u_{i+\frac{1}{2}}$, even at intervals located close to a discontinuity. 
Proposition 5.3. Let $\alpha:=\max _{x \in \Omega}\left|\frac{u^{(i v)}(x)}{3 u(x)}\right|$. Assume $\alpha<+\infty$ and define

$$
\varepsilon_{\text {alt }}^{4}:=\operatorname{sign}\left(\varepsilon_{\text {lin }}^{4}\right) \min \left(a b s\left(\varepsilon_{\text {lin }}^{4}\right), c \alpha\right), \quad c>1
$$

Then:

1. If $u$ is smooth at $\left[x_{i-2}, x_{i+3}\right]$, then $u_{i+\frac{1}{2}}-G_{4}^{W E N O}\left(x_{i+\frac{1}{2}}, \varepsilon_{a l t}\right)=O\left(h^{5}\right)$.

2. If $u$ has an isolated discontinuity at $\left[x_{i-2}, x_{i}\right]\left(\right.$ or $\left.\left[x_{i+1}, x_{i+3}\right]\right)$, then $u_{i+\frac{1}{2}}-G_{4}^{W E N O}\left(x_{i+\frac{1}{2}}, \varepsilon_{a l t}\right)=$ $O\left(h^{3}\right)$.

Proof. Observe that, from (50), $u_{i+\frac{1}{2}}-G_{4}\left(x_{i+\frac{1}{2}}, C\right)=O\left(h^{3}\right)$ for any constant $C$ if there is a discontinuity located at $\left[x_{i-2}, x_{i}\right]$ or $\left[x_{i+1}, x_{i+3}\right]$.

Lastly, we show that it is possible to compute the shape parameter in a WENO-like adaptive manner and improve the approximation error at the intervals adjacent to a jump discontinuity. For this we need to define one-sided approximations to $u_{i+\frac{1}{2}}^{(i v)}$, and combine them using appropriate WENO-type weights that ensure that, when a discontinuity is close to $x_{i+\frac{1}{2}}$, at least one of the approximations remains accurate. Consider the following first-order, one-sided approximations to $u_{i+\frac{1}{2}}^{(i v)}$

$$
\begin{aligned}
& u_{i+\frac{1}{2}, L}^{(i v)}:=\frac{1}{h^{4}}\left(u_{i-3}-4 u_{i-2}+6 u_{i-1}-4 u_{i}+u_{i+1}\right), \\
& u_{i+\frac{1}{2}, R}^{(i v)}:=\frac{1}{h^{4}}\left(u_{i}-4 u_{i+1}+6 u_{i+2}-4 u_{i+3}+u_{i+4}\right),
\end{aligned}
$$

and the following WENO-type weights

$$
\omega_{5}^{i, b}=\frac{\alpha_{5}^{i, b}}{\alpha_{5}^{i, 1}+\alpha_{5}^{i, 4}}, \quad \alpha_{5}^{i, b}=\frac{1 / 2}{\left(h^{2}+I_{5}^{i, b}\right)^{3}}, \quad b=1,4,
$$

where

$$
\begin{aligned}
& I_{5}^{i, 1}=\left(u_{i-3}-4 u_{i-2}+6 u_{i-1}-4 u_{i}+u_{i+1}\right)^{2} \\
& I_{5}^{i, 4}=\left(u_{i}-4 u_{i+1}+6 u_{i+2}-4 u_{i+3}+u_{i+4}\right)^{2} .
\end{aligned}
$$

Note that in smooth regions $u_{i+\frac{1}{2}, L, R}^{(i v)}=u_{i+\frac{1}{2}}^{(i v)}+O(h)$, but if the stencil chosen to compute the approximation crosses a jump discontinuity, then $u_{i+\frac{1}{2}, L, R}^{(i v)}=O\left(h^{-4}\right)$.

We define

$$
\varepsilon_{w e n}^{4}:=-\frac{\omega_{5}^{i, 1} u_{i+\frac{1}{2}, L}^{(i v)}+\omega_{5}^{i, 4} u_{i+\frac{1}{2}, R}^{(i v)}}{\frac{3}{2}\left(u_{i}+u_{i+1}\right)} .
$$

Then we have the following results.

Proposition 5.4. $\varepsilon_{w e n}^{4}$ in (53) satisfies the following.

1. If $u \in C^{5}\left(\left[x_{i-3}, x_{i+2}\right]\right)$ or $u \in C^{5}\left(\left[x_{i-1}, x_{i+4}\right]\right)$ then

$$
\varepsilon_{w e n}^{4}=\varepsilon_{o p t}^{4}+O(h) \Rightarrow u_{i+\frac{1}{2}}-G_{4}^{W E N O}\left(x_{i+\frac{1}{2}}, \varepsilon_{w e n}\right)=O\left(h^{5}\right) .
$$

2. If $u$ has a discontinuity at $\left[x_{i-2}, x_{i}\right]$ (or $\left.\left[x_{i+1}, x_{i+3}\right]\right)$ and is smooth in the rest of the domain, then

$$
\varepsilon_{w e n}^{4}=\varepsilon_{o p t}^{4}+O(h) \quad \Rightarrow \quad u_{i+\frac{1}{2}}-G_{4}^{W E N O}\left(x_{i+\frac{1}{2}}, \varepsilon_{w e n}\right)=O\left(h^{3}\right) .
$$

Proof. 
1. If $u \in C^{5}\left(\left[x_{i-3}, x_{i+4}\right]\right)$ then

$$
u_{i+\frac{1}{2}, L, R}^{(i v)}=u_{i+\frac{1}{2}}^{(i v)}+O(h), \quad \omega_{5}^{i, 1}=\frac{1}{2}+O\left(h^{2}\right), \quad \omega_{5}^{i, 4}=\frac{1}{2}+O\left(h^{2}\right)
$$

hence

$$
\varepsilon_{w e n}^{4}=\frac{u_{i+\frac{1}{2}}^{(i v)}+O(h)}{3 u_{i+\frac{1}{2}}+O\left(h^{2}\right)}=\varepsilon_{o p t}^{4}+O(h) .
$$

Since

$$
u_{i+\frac{1}{2}}-G_{4}^{W E N O}\left(x_{i+\frac{1}{2}}, \varepsilon\right)=\frac{3}{128}\left(u_{i+\frac{1}{2}}^{(i v)}+3 \varepsilon^{4} u_{i+\frac{1}{2}}\right) h^{4}+O\left(h^{5}\right),
$$

we get the desired result.

Now, if $u \in C^{5}\left(\left[x_{i-3}, x_{i+2}\right]\right)$ but has an isolated discontinuity at $\left[x_{i+2}, x_{i+4}\right]$, we have

$$
u_{i+\frac{1}{2}, L}^{(i v)}=u_{i+\frac{1}{2}}^{(i v)}+O(h), \quad u_{i+\frac{1}{2}, R}^{(i v)}=O\left(h^{-4}\right),
$$

but using Taylor expansions we can prove that

$$
\omega_{5}^{i, 1} u_{i+\frac{1}{2}, L}^{(i v)}+\omega_{5}^{i, 4} u_{i+\frac{1}{2}, R}^{(i v)}=u_{i+\frac{1}{2}}^{(i v)}+O(h)
$$

and the result also follows from the expression of the error above.

2. Assume that $u$ has a discontinuity at $\left[x_{i-3}, x_{i-1}\right]$. Then

$$
u_{i+\frac{1}{2}, R}^{(i v)}=u_{i+\frac{1}{2}}^{(i v)}+O(h), \quad u_{i+\frac{1}{2}, L}^{(i v)}=O\left(h^{-4}\right), \quad \omega_{5}^{i, 1}=O\left(h^{6}\right), \quad \omega_{5}^{i, 4}=1+O\left(h^{6}\right)
$$

and

$$
\omega_{5}^{i, 1} u_{i+\frac{1}{2}, L}^{(i v)}+\omega_{5}^{i, 4} u_{i+\frac{1}{2}, R}^{(i v)}=u_{i+\frac{1}{2}}^{(i v)}+O(h)
$$

The result follows from (50).

\subsection{Accuracy of $R_{4}^{W E N O}\left(x_{i+\frac{1}{2}}, \varepsilon\right)$}

We examine the effect on the overall accuracy of the two different estimations of the shape parameter considered in Section 4.2. Propositions 4.8, 4.9 and 4.10 can be directly generalized to the case considered here.

Proposition 5.5. Let $\varepsilon_{\text {lin }}^{2}$ be defined as in (38).

1. If $u \in C^{5}\left(\left[x_{i-1}, x_{i+2}\right]\right)$, then $u_{i+\frac{1}{2}}-R_{4}^{W E N O}\left(x_{i+\frac{1}{2}}, \varepsilon_{l i n}\right)=O\left(h^{4}\right)$.

2. If $u$ has a discontinuity at $\left[x_{i-1}, x_{i}\right]$ (or $\left.\left[x_{i+1}, x_{i+2}\right]\right)$, then $u_{i+\frac{1}{2}}-R_{4}^{W E N O}\left(x_{i+\frac{1}{2}}, \varepsilon_{l i n}\right)=O\left(\frac{1}{h^{2}}\right)$.

Proposition 5.6. Let $\varepsilon_{\text {alt }}^{2}$ be defined as in (39).

1. If $u$ is smooth in $\left[x_{i-1}, x_{i+2}\right]$, then $u_{i+\frac{1}{2}}-R_{4}^{W E N O}\left(x_{i+\frac{1}{2}}, \varepsilon_{\text {alt }}\right)=O\left(h^{4}\right)$.

2. If $u$ has a discontinuity at $\left[x_{i-1}, x_{i}\right]$ (or $\left.\left[x_{i+1}, x_{i+2}\right]\right)$, then $u_{i+\frac{1}{2}}-R_{4}^{W E N O}\left(x_{i+\frac{1}{2}}, \varepsilon_{\text {alt }}\right)=O\left(h^{3}\right)$.

In light of Proposition 5.5, the approximation of $u_{i+\frac{1}{2}}$ obtained from $R_{4}^{W E N O}\left(x_{i+\frac{1}{2}}, \varepsilon_{\text {lin }}\right)$ at intervals located next to a jump discontinuity is extremely bad. The reason is, of course, that the stencil of points used to construct $\varepsilon_{l i n}$ crosses the discontinuity, hence $\varepsilon_{l i n}=O\left(h^{-4}\right)$. As done in Section 4.1, we can avoid this situation by using a WENO-type adaptive nonlinear estimation of the optimal shape parameter. For this, it is sufficient to consider one-sided first-order estimates of $u_{i+\frac{1}{2}}^{\prime \prime \prime}$ and appropriate WENO-type weights. Thus, we consider 


$$
\begin{aligned}
& u_{i+\frac{1}{2}, L}^{\prime \prime \prime}:=\frac{1}{h^{3}}\left(-u_{i-2}+3 u_{i-1}-3 u_{i}+u_{i+1}\right), \\
& u_{i+\frac{1}{2}, R}^{\prime \prime \prime}:=\frac{1}{h^{3}}\left(-u_{i}+3 u_{i+1}-3 u_{i+2}+u_{i+3}\right),
\end{aligned}
$$

and define

$$
\varepsilon_{w e n}^{2}:=-\frac{\omega_{4}^{i, 1} u_{i+\frac{1}{2}}^{\prime \prime \prime}+\omega_{4}^{i, 3} u_{i+\frac{1}{2}}^{\prime \prime \prime}}{3\left(u_{i+1}-u_{i}\right) / h}
$$

where

with

$$
\omega_{4}^{i, b}=\frac{\alpha_{4}^{i, b}}{\alpha_{4}^{i, 1}+\alpha_{4}^{i, 3}}, \quad \alpha_{4}^{i, b}=\frac{1 / 2}{\left(h^{2}+I_{4}^{i, b}\right)^{3}}, \quad b=1,3
$$

$$
I_{4}^{i, b}=\left(-u_{i+b-3}+3 u_{i+b-2}-3 u_{i+b-1}+u_{i+b}\right)^{2}, \quad b=1,3
$$

denoting the smoothness indicators of the function $u$ in the intervals $\left[x_{i+b-4}, x_{i+b}\right], b=1,3$.

Then we have the following result.

Proposition 5.7. Let $\varepsilon_{\text {wen }}^{2}$ be defined as in (54).

1. If $u \in C^{5}\left(\left[x_{i-1}, x_{i+3}\right]\right)$ or $u \in C^{5}\left(\left[x_{i-2}, x_{i+2}\right]\right)$ then

$$
\varepsilon_{w e n}^{2}=\varepsilon_{o p t}^{2}+O(h) \quad \text { and } \quad u_{i+\frac{1}{2}}-R_{4}^{W E N O}\left(x_{i+\frac{1}{2}}, \varepsilon_{w e n}\right)=O\left(h^{4}\right) .
$$

2. If $u$ has an isolated discontinuity at $\left[x_{i-1}, x_{i}\right]$ (or $\left.\left[x_{i+1}, x_{i+2}\right]\right)$ and is smooth in $\left[x_{i}, x_{i+3}\right]$ (or in $\left.\left[x_{i-2}, x_{i+1}\right]\right)$, then

$$
\varepsilon_{w e n}^{2}=\varepsilon_{o p t}^{2}+O(h) \quad \text { and } \quad u_{i+\frac{1}{2}}-R_{4}^{W E N O}\left(x_{i+\frac{1}{2}}, \varepsilon_{w e n}\right)=O\left(h^{4}\right) .
$$

In the next section we show, through numerical experiments, that the theoretical orders of accuracy of the different MQ-RBF-WENO reconstructions proposed and analyzed in this paper are recovered in practice. We summarize in Table 1 the results of previous Sections to facilitate the comparisons.

\begin{tabular}{|c|c|c|c|c|c|c|}
\hline \multirow{2}{*}{} & \multicolumn{2}{|c|}{$u_{i+\frac{1}{2}}-\bar{R}_{2}^{i, 1}\left(x_{i+\frac{1}{2}}, \varepsilon\right)$} & \multicolumn{2}{|c|}{$u_{i+\frac{1}{2}}-R_{4}^{W E N O}\left(x_{i+\frac{1}{2}}, \varepsilon\right)$} & \multicolumn{2}{|c|}{$u_{i+\frac{1}{2}}-G_{4}^{W E N O}\left(x_{i+\frac{1}{2}}, \varepsilon\right)$} \\
\cline { 2 - 7 } & smooth & disc & smooth & disc & smooth & disc \\
\hline$\varepsilon_{\text {opt }}$ & $O\left(h^{4}\right)$ & $O\left(h^{4}\right)$ & $O\left(h^{4}\right)$ & $O\left(h^{4}\right)$ & $O\left(h^{5}\right)$ & $O\left(h^{3}\right)$ \\
\hline$\varepsilon_{\text {lin }}$ & $O\left(h^{4}\right)$ & $O(1)$ & $O\left(h^{4}\right)$ & $O\left(h^{-2}\right)$ & $O\left(h^{5}\right)$ & $O(1)$ \\
\hline$\varepsilon_{\text {alt }}$ & $O\left(h^{4}\right)$ & $O\left(h^{2}\right)$ & $O\left(h^{4}\right)$ & $O\left(h^{3}\right)$ & $O\left(h^{5}\right)$ & $O\left(h^{3}\right)$ \\
\hline$\varepsilon_{\text {wen }}$ & $O\left(h^{4}\right)$ & $O\left(h^{3}\right)$ & $O\left(h^{4}\right)$ & $O\left(h^{4}\right)$ & $O\left(h^{5}\right)$ & $O\left(h^{3}\right)$ \\
\hline
\end{tabular}

Table 1: Orders of accuracy for different reconstructions and different $\varepsilon$. The term smooth refers to the function $u$ being as smooth as required by the theoretical Propositions. The term disc refers to the function being smooth in $\left[x_{i}, x_{i+1}\right]$, with an isolated discontinuity in an interval adjacent to $\left[x_{i}, x_{i+1}\right]$.

Remark 5.8. In smooth regions $R_{4}^{W E N O}\left(x_{i+\frac{1}{2}}, \varepsilon\right)$ provides a fourth-order approximation of $u_{i+\frac{1}{2}}$, for $\varepsilon=$ $\varepsilon_{\text {lin }}$ in (38), i.e., the required computations involve the stencil $\left\{x_{i-1}, x_{i}, x_{i+1}, x_{i+2}\right\}$. However, it should be remarked that this approximation becomes extremely bad near jump discontinuities, and an adaptive computation of the shape parameter becomes mandatory (see next Section also). Note that $G_{4}^{W E N O}\left(x_{i+\frac{1}{2}}, \varepsilon\right)$, for $\varepsilon=\varepsilon_{\text {lin }}$ in (48) becomes fifth-order accurate, with a poor approximation near jumps (just as in the case of the 2-point MQ-RBF interpolant, with a linear estimate of the shape parameter). The accuracy may be improved, without reverting to a WENO polynomial approximation, by using a WENO-adaptive computation of the shape parameter. Note that $\varepsilon_{\text {wen }}$ is the estimate that matches the accuracy order provided by $\varepsilon_{\text {opt }}$ both in smooth regions and near a discontinuity when used with $G_{4}^{W E N O}$ and $R_{4}^{W E N O}$, respectively. 


\section{Numerical experiments: reconstruction of discontinuous functions in 1-D}

The main goal of this section is to validate the theoretical results on the approximation order of the MQ-RBF and MQ-RBF-WENO reconstruction methods proposed in the previous Sections.

To this end we consider the discontinuous test function $u:[0,1] \rightarrow \mathbb{R}$, piecewisely defined as

$$
u(x)=\left\{\begin{array}{ccc}
e^{(x-0.5)} & \text { if } \quad 0 \leq x \leq 0.5 \\
1+e^{(x-0.5)} & \text { if } \quad 0.5<x \leq 1
\end{array}\right.
$$

Starting from its samples $\left\{u_{i}^{\ell}=u\left(x_{i}^{\ell}\right)\right\}_{i=0, \ldots, 2^{\ell}}, \ell=6,7, \ldots, 13$, obtained by evaluating $u$ at the abscissa values $x_{i}^{\ell}=\frac{i}{2^{\ell}}$, we can use the previously presented strategies as prediction methods to compute the approximated values $\left\{\hat{u}_{i}^{\ell+1}\right\}_{i=0, \ldots, 2^{\ell+1}}$ to the samples $\left\{u_{i}^{\ell+1}=u\left(x_{i}^{\ell+1}\right)\right\}_{i=0, \ldots, 2^{\ell+1}}$.

Let $\Omega_{d}:=[0, d] \subset \Omega=[0,1]$, and define

$$
e_{\ell}:=\max \left\{\left|u\left(x_{i+\frac{1}{2}}^{\ell}\right)-\hat{u}_{2 i+1}^{\ell+1}\right|, x_{2 i+1}^{\ell+1}=x_{i+\frac{1}{2}}^{\ell}=\frac{x_{i}^{\ell}+x_{i+1}^{\ell}}{2} \in \Omega_{d}\right\}
$$

with $\hat{u}_{2 i+1}^{\ell+1}$ the approximation obtained with the reconstruction techniques in Table 1 . If $e_{\ell} \approx\left(h_{\ell}\right)^{p}$ with $h_{\ell}=x_{i+1}^{\ell}-x_{i}^{\ell}=\frac{1}{2^{\ell}}$, then $\log _{2}\left(e_{\ell-1} / e_{\ell}\right) \approx p$, and thus $\log _{2}\left(e_{\ell-1} / e_{\ell}\right)$ can be assumed as an estimation of the approximation order.

\subsection{Smooth regions: far from jump discontinuities}

For $d=0.25$, we have that $u$ is smooth in a domain that contains $\Omega_{0.25}$ and it is sufficiently well separated from the jump discontinuity (located at 0.5).

The numerical results collected in Table 2 show that, in a smooth region, we obtain approximation order 4 when using $\bar{R}_{2}^{i, 1}$ with the estimation of the shape parameter provided by $\varepsilon_{\text {lin }}^{2}$ in (32), $\varepsilon_{\text {alt }}^{2}$ in (33) and $\varepsilon_{\text {wen }}^{2}$ in (36) (according exactly to the theoretical results in Propositions 4.2, 4.4 and 4.6).

The numerical results collected in Table 3 show the improved approximation order 4 for the reconstruction technique $R_{4}^{W E N O}$ using $\varepsilon_{\text {lin }}^{2}$ in (38), $\varepsilon_{\text {alt }}^{2}$ in (39) and $\varepsilon_{w e n}^{2}$ in (54). This is in full agreement with the theoretical results in Propositions 5.5, 5.6 and 5.7.

Likewise, the numerical results collected in Table 4 show the improved approximation order 5 for the reconstruction technique $G_{4}^{W E N O}$ using $\varepsilon_{\text {lin }}^{4}$ in (48), $\varepsilon_{\text {alt }}^{4}$ in (51) and $\varepsilon_{\text {wen }}^{4}$ in (53). This is in full agreement with the theoretical results in Propositions 5.2, 5.3 and 5.4.

\begin{tabular}{|c|c|c|c|c|c|c|}
\hline \multirow{3}{*}{$\ell$} & \multicolumn{5}{|c|}{$\Omega_{0.25}=[0,0.25]$} \\
\cline { 2 - 7 } & $u_{i+\frac{1}{2}}-\bar{R}_{2}^{\imath, 1}\left(x_{i+\frac{1}{2}}^{\ell}, \varepsilon_{l i n}\right)$ & \multicolumn{2}{c|}{$u_{i+\frac{1}{2}}-\bar{R}_{2}^{2,1}\left(x_{i+\frac{1}{2}}^{\ell}, \varepsilon_{a l t}\right)$} & \multicolumn{2}{c|}{$u_{i+\frac{1}{2}}-\bar{R}_{2}^{\imath, 1}\left(x_{i+\frac{1}{2}}^{\ell}, \varepsilon_{w e n}\right)$} \\
\cline { 2 - 7 } & $e_{\ell}$ & $\log _{2}\left(e_{\ell-1} / e_{\ell}\right)$ & $e_{\ell}$ & $\log _{2}\left(e_{\ell-1} / e_{\ell}\right)$ & $e_{\ell}$ & $\log _{2}\left(e_{\ell-1} / e_{\ell}\right)$ \\
\hline 6 & $2.8783 \mathrm{e}-09$ & $3.9942 \mathrm{e}+00$ & $2.8783 \mathrm{e}-09$ & $3.9942 \mathrm{e}+00$ & $2.8957 \mathrm{e}-09$ & $3.9986 \mathrm{e}+00$ \\
7 & $1.8062 \mathrm{e}-10$ & $3.9971 \mathrm{e}+00$ & $1.8062 \mathrm{e}-10$ & $3.9971 \mathrm{e}+00$ & $1.8115 \mathrm{e}-10$ & $3.9993 \mathrm{e}+00$ \\
8 & $1.1311 \mathrm{e}-11$ & $3.9986 \mathrm{e}+00$ & $1.1311 \mathrm{e}-11$ & $3.9986 \mathrm{e}+00$ & $1.1328 \mathrm{e}-11$ & $3.9997 \mathrm{e}+00$ \\
9 & $7.0762 \mathrm{e}-13$ & $3.9993 \mathrm{e}+00$ & $7.0762 \mathrm{e}-13$ & $3.9993 \mathrm{e}+00$ & $7.0815 \mathrm{e}-13$ & $3.9998 \mathrm{e}+00$ \\
10 & $4.4248 \mathrm{e}-14$ & $3.9996 \mathrm{e}+00$ & $4.4248 \mathrm{e}-14$ & $3.9996 \mathrm{e}+00$ & $4.4264 \mathrm{e}-14$ & $3.9999 \mathrm{e}+00$ \\
11 & $2.7662 \mathrm{e}-15$ & $3.9998 \mathrm{e}+00$ & $2.7662 \mathrm{e}-15$ & $3.9998 \mathrm{e}+00$ & $2.7667 \mathrm{e}-15$ & $4.0000 \mathrm{e}+00$ \\
12 & $1.7291 \mathrm{e}-16$ & $3.9999 \mathrm{e}+00$ & $1.7291 \mathrm{e}-16$ & $3.9999 \mathrm{e}+00$ & $1.7292 \mathrm{e}-16$ & $4.0000 \mathrm{e}+00$ \\
13 & $1.0807 \mathrm{e}-17$ & $4.0000 \mathrm{e}+00$ & $1.0807 \mathrm{e}-17$ & $4.0000 \mathrm{e}+00$ & $1.0808 \mathrm{e}-17$ & $4.0000 \mathrm{e}+00$ \\
\hline
\end{tabular}

Table 2: Smooth region, far from jumps: errors $e_{\ell}$ in (57) and estimates of the approximation order for $\hat{u}_{2 i+1}^{\ell+1}=\bar{R}_{2}^{i, 1}\left(x_{i+\frac{1}{2}}^{\ell}, \varepsilon\right)$ with $\varepsilon_{\text {lin }}^{2}$ in (32) (left), $\varepsilon_{\text {alt }}^{2}$ in (33) (center) and $\varepsilon_{w e n}^{2}$ in (36) (right). 


\begin{tabular}{|c|c|c|c|c|c|c|}
\hline \multirow{3}{*}{$\ell$} & \multicolumn{6}{|c|}{$\Omega_{0.25}=[0,0.25]$} \\
\cline { 2 - 7 } & $u_{i+\frac{1}{2}}-R_{4}^{W E N O}\left(x_{i+\frac{1}{2}}^{\ell}, \varepsilon_{l i n}\right)$ & $u_{i+\frac{1}{2}}-R_{4}^{W E N O}\left(x_{i+\frac{1}{2}}^{\ell}, \varepsilon_{a l t}\right)$ & $u_{i+\frac{1}{2}}-R_{4}^{W E N O}\left(x_{i+\frac{1}{2}}^{\ell}, \varepsilon_{w e n}\right)$ \\
\cline { 2 - 7 } & $e_{\ell}$ & $\log _{2}\left(e_{\ell-1} / e_{\ell}\right)$ & $e_{\ell}$ & $\log _{2}\left(e_{\ell-1} / e_{\ell}\right)$ & $e_{\ell}$ & $\log _{2}\left(e_{\ell-1} / e_{\ell}\right)$ \\
\hline 6 & $1.4394 \mathrm{e}-09$ & $3.9944 \mathrm{e}+00$ & $1.4394 \mathrm{e}-09$ & $3.9944 \mathrm{e}+00$ & $1.4394 \mathrm{e}-09$ & $3.9944 \mathrm{e}+00$ \\
7 & $9.0311 \mathrm{e}-11$ & $3.9972 \mathrm{e}+00$ & $9.0311 \mathrm{e}-11$ & $3.9972 \mathrm{e}+00$ & $9.0313 \mathrm{e}-11$ & $3.9972 \mathrm{e}+00$ \\
8 & $5.6555 \mathrm{e}-12$ & $3.9986 \mathrm{e}+00$ & $5.6555 \mathrm{e}-12$ & $3.9986 \mathrm{e}+00$ & $5.6555 \mathrm{e}-12$ & $3.9986 \mathrm{e}+00$ \\
9 & $3.5381 \mathrm{e}-13$ & $3.9993 \mathrm{e}+00$ & $3.5381 \mathrm{e}-13$ & $3.9993 \mathrm{e}+00$ & $3.5381 \mathrm{e}-13$ & $3.9993 \mathrm{e}+00$ \\
10 & $2.2124 \mathrm{e}-14$ & $3.9996 \mathrm{e}+00$ & $2.2124 \mathrm{e}-14$ & $3.9996 \mathrm{e}+00$ & $2.2124 \mathrm{e}-14$ & $3.9996 \mathrm{e}+00$ \\
11 & $1.3831 \mathrm{e}-15$ & $3.9998 \mathrm{e}+00$ & $1.3831 \mathrm{e}-15$ & $3.9998 \mathrm{e}+00$ & $1.3831 \mathrm{e}-15$ & $3.9998 \mathrm{e}+00$ \\
12 & $8.6454 \mathrm{e}-17$ & $3.9999 \mathrm{e}+00$ & $8.6454 \mathrm{e}-17$ & $3.9999 \mathrm{e}+00$ & $8.6454 \mathrm{e}-17$ & $3.9999 \mathrm{e}+00$ \\
13 & $5.4037 \mathrm{e}-18$ & $4.0000 \mathrm{e}+00$ & $5.4037 \mathrm{e}-18$ & $4.0000 \mathrm{e}+00$ & $5.4037 \mathrm{e}-18$ & $4.0000 \mathrm{e}+00$ \\
\hline
\end{tabular}

Table 3: Smooth region, far from jumps: errors $e_{\ell}$ in (57) and estimates of the approximation order for $\hat{u}_{2 i+1}^{\ell+1}=R_{4}^{W E N O}\left(x_{i+\frac{1}{2}}^{\ell}, \varepsilon\right)$ with $\varepsilon_{\text {lin }}^{2}$ in (38) (left), $\varepsilon_{\text {alt }}^{2}$ in (39) (center) and $\varepsilon_{w e n}^{2}$ in (54) (right).

\begin{tabular}{|c|c|c|c|c|c|c|}
\hline \multirow{3}{*}{$\ell$} & \multicolumn{6}{|c|}{$\Omega_{0.25}=[0,0.25]$} \\
\cline { 2 - 7 } & $u_{i+\frac{1}{2}}-G_{4}^{W E N O}\left(x_{i+\frac{1}{2}}^{\ell}, \varepsilon_{l i n}\right)$ & $u_{i+\frac{1}{2}}-G_{4}^{W E N O}\left(x_{i+\frac{1}{2}}^{\ell}, \varepsilon_{a l t}\right)$ & $u_{i+\frac{1}{2}}-G_{4}^{W E N O}\left(x_{i+\frac{1}{2}}^{\ell}, \varepsilon_{w e n}\right)$ \\
\cline { 2 - 7 } & $e_{\ell}$ & $\log _{2}\left(e_{\ell-1} / e_{\ell}\right)$ & $e_{\ell}$ & $\log _{2}\left(e_{\ell-1} / e_{\ell}\right)$ & $e_{\ell}$ & $\log _{2}\left(e_{\ell-1} / e_{\ell}\right)$ \\
\hline 6 & $1.7380 \mathrm{e}-11$ & $5.0110 \mathrm{e}+00$ & $1.7380 \mathrm{e}-11$ & $5.0110 \mathrm{e}+00$ & $1.7644 \mathrm{e}-11$ & $5.0217 \mathrm{e}+00$ \\
7 & $5.3900 \mathrm{e}-13$ & $5.0056 \mathrm{e}+00$ & $5.3900 \mathrm{e}-13$ & $5.0056 \mathrm{e}+00$ & $5.4313 \mathrm{e}-13$ & $5.0110 \mathrm{e}+00$ \\
8 & $1.6779 \mathrm{e}-14$ & $5.0028 \mathrm{e}+00$ & $1.6779 \mathrm{e}-14$ & $5.0028 \mathrm{e}+00$ & $1.6844 \mathrm{e}-14$ & $5.0056 \mathrm{e}+00$ \\
9 & $5.2332 \mathrm{e}-16$ & $5.0014 \mathrm{e}+00$ & $5.2332 \mathrm{e}-16$ & $5.0014 \mathrm{e}+00$ & $5.2433 \mathrm{e}-16$ & $5.0028 \mathrm{e}+00$ \\
10 & $1.6338 \mathrm{e}-17$ & $5.0007 \mathrm{e}+00$ & $1.6338 \mathrm{e}-17$ & $5.0007 \mathrm{e}+00$ & $1.6354 \mathrm{e}-17$ & $5.0014 \mathrm{e}+00$ \\
11 & $5.1031 \mathrm{e}-19$ & $5.0004 \mathrm{e}+00$ & $5.1031 \mathrm{e}-19$ & $5.0004 \mathrm{e}+00$ & $5.1056 \mathrm{e}-19$ & $5.0007 \mathrm{e}+00$ \\
12 & $1.5943 \mathrm{e}-20$ & $5.0002 \mathrm{e}+00$ & $1.5943 \mathrm{e}-20$ & $5.0002 \mathrm{e}+00$ & $1.5947 \mathrm{e}-20$ & $5.0004 \mathrm{e}+00$ \\
13 & $4.9817 \mathrm{e}-22$ & $5.0001 \mathrm{e}+00$ & $4.9817 \mathrm{e}-22$ & $5.0001 \mathrm{e}+00$ & $4.9823 \mathrm{e}-22$ & $5.0002 \mathrm{e}+00$ \\
\hline
\end{tabular}

Table 4: Smooth region, far from jumps: errors $e_{\ell}$ in (57) and estimates of the approximation order for $\hat{u}_{2 i+1}^{\ell+1}=G_{4}^{W E N O}\left(x_{i+\frac{1}{2}}^{\ell}, \varepsilon\right)$ with $\varepsilon_{l i n}^{4}$ in (48) (left), $\varepsilon_{a l t}^{4}$ in (51) (center) and $\varepsilon_{w e n}^{4}$ in (53) (right).

\subsection{Smooth regions: next to a jump discontinuity}

The function in (56) is smooth in $[0,0.5)$, but since the jump discontinuity is located precisely at 0.5 , the error in (57) for $\Omega_{0.5}$ will be dominated by the larger error obtained at the interval located next to the jump discontinuity in $u$.

Tables 5, 6 and 7 collect the results obtained from computing the error in (57) for the reconstruction techniques $\bar{R}_{2}^{i, 1}, R_{4}^{W E N O}$ and $G_{4}^{W E N O}$, respectively.

Note that the approximation orders obtained in the theoretical results of the previous Sections are numerically confirmed by these tables. In particular, for $\bar{R}_{2}^{i, 1}$ and $G_{4}^{W E N O}, \log _{2}\left(e_{\ell-1} / e_{\ell}\right) \approx 0$, i.e. the approximation order is thus only 1, as stated in Propositions 4.3 and 5.2. We also note in Table 6 that when we use $\varepsilon_{\text {lin }}^{2}$ in $R_{4}^{W E N O}$ we obtain an approximation of order -2 which agrees with Proposition 5.5.

It is worth noting that, with very little work with respect to the computation of the linear optimal shape parameter, the order of approximation at intervals close to a discontinuity can be improved by considering the nonlinear approximations $\varepsilon_{\text {alt }}^{2}$ and $\varepsilon_{\text {alt }}^{4}$ in (39) and (51). For $\bar{R}_{2}^{i, 1}$ we obtain an approximation order 2 (in agreement with Proposition 4.4), for $R_{4}^{W E N O}$ an approximation order 3 (in agreement with Proposition 5.6) and for $G_{4}^{W E N O}$ an approximation order 3 (in agreement with Proposition 5.3).

Finally, the nonlinear approximations $\varepsilon_{w e n}^{2}$ and $\varepsilon_{w e n}^{4}$ provide an approximation order 3 for $\bar{R}_{2}^{i, 1}$, an approximation order 4 for $R_{4}^{W E N O}$ and an approximation order 3 for $G_{4}^{W E N O}$ (in agreement respectively with Propositions 4.6, 5.7 and 5.4). 


\begin{tabular}{|c|c|c|c|c|c|c|}
\hline \multirow{3}{*}{$\ell$} & \multicolumn{5}{|c|}{$\Omega_{0.5}=[0,0.5]$} \\
\cline { 2 - 7 } & $u_{i+\frac{1}{2}}-\bar{R}_{2}^{\imath, 1}\left(x_{i+\frac{1}{2}}^{\ell}, \varepsilon_{l i n}\right)$ & \multicolumn{2}{|c|}{$u_{i+\frac{1}{2}}-\bar{R}_{2}^{i, 1}\left(x_{i+\frac{1}{2}}^{\ell}, \varepsilon_{a l t}\right)$} & \multicolumn{2}{|c|}{$u_{i+\frac{1}{2}}-\bar{R}_{2}^{i, 1}\left(x_{i+\frac{1}{2}}^{\ell}, \varepsilon_{w e n}\right)$} \\
\cline { 2 - 7 } & $e_{\ell}$ & $\log _{2}\left(e_{\ell-1} / e_{\ell}\right)$ & $e_{\ell}$ & $\log _{2}\left(e_{\ell-1} / e_{\ell}\right)$ & $e_{\ell}$ & $\log _{2}\left(e_{\ell-1} / e_{\ell}\right)$ \\
\hline 6 & $4.8401 \mathrm{e}-02$ & $-9.7992 \mathrm{e}-04$ & $6.0517 \mathrm{e}-05$ & $1.9936 \mathrm{e}+00$ & $2.2384 \mathrm{e}-07$ & $2.9543 \mathrm{e}+00$ \\
7 & $4.8434 \mathrm{e}-02$ & $-4.3687 \mathrm{e}-04$ & $1.5197 \mathrm{e}-05$ & $1.9970 \mathrm{e}+00$ & $2.8881 \mathrm{e}-08$ & $2.9773 \mathrm{e}+00$ \\
8 & $4.8448 \mathrm{e}-02$ & $-2.0507 \mathrm{e}-04$ & $3.8071 \mathrm{e}-06$ & $1.9985 \mathrm{e}+00$ & $3.6674 \mathrm{e}-09$ & $2.9887 \mathrm{e}+00$ \\
9 & $4.8455 \mathrm{e}-02$ & $-9.9179 \mathrm{e}-05$ & $9.5273 \mathrm{e}-07$ & $1.9993 \mathrm{e}+00$ & $4.6203 \mathrm{e}-10$ & $2.9944 \mathrm{e}+00$ \\
10 & $4.8459 \mathrm{e}-02$ & $-4.8750 \mathrm{e}-05$ & $2.3830 \mathrm{e}-07$ & $1.9996 \mathrm{e}+00$ & $5.7981 \mathrm{e}-11$ & $2.9972 \mathrm{e}+00$ \\
11 & $4.8460 \mathrm{e}-02$ & $-2.4165 \mathrm{e}-05$ & $5.9590 \mathrm{e}-08$ & $1.9998 \mathrm{e}+00$ & $7.2618 \mathrm{e}-12$ & $2.9986 \mathrm{e}+00$ \\
12 & $4.8461 \mathrm{e}-02$ & $-1.2030 \mathrm{e}-05$ & $1.4899 \mathrm{e}-08$ & $1.9999 \mathrm{e}+00$ & $9.0861 \mathrm{e}-13$ & $2.9993 \mathrm{e}+00$ \\
13 & $4.8462 \mathrm{e}-02$ & $-6.0018 \mathrm{e}-06$ & $3.7251 \mathrm{e}-09$ & $2.0000 \mathrm{e}+00$ & $1.1363 \mathrm{e}-13$ & $2.9996 \mathrm{e}+00$ \\
\hline
\end{tabular}

Table 5: Smooth region, next to a jump: errors $e_{\ell}$ in (57) and estimates of the approximation order for $\hat{u}_{2 i+1}^{\ell+1}=\bar{R}_{2}^{i, 1}\left(x_{i+\frac{1}{2}}^{\ell}, \varepsilon\right)$ with $\varepsilon_{l i n}^{2}$ in (32) (left), $\varepsilon_{a l t}^{2}$ in (33) (center) and $\varepsilon_{w e n}^{2}$ in (36) (right).

\begin{tabular}{|c|c|c|c|c|c|c|}
\hline \multirow{3}{*}{$\ell$} & \multicolumn{5}{|c|}{$\Omega_{0.5}=[0,0.5]$} \\
\cline { 2 - 7 } & $u_{i+\frac{1}{2}}-R_{4}^{W E N O}\left(x_{i+\frac{1}{2}}^{\ell}, \varepsilon_{l i n}\right)$ & $u_{i+\frac{1}{2}}-R_{4}^{W E N O}\left(x_{i+\frac{1}{2}}^{\ell}, \varepsilon_{a l t}\right)$ & $u_{i+\frac{1}{2}}-R_{4}^{W E N O}\left(x_{i+\frac{1}{2}}^{\ell}, \varepsilon_{w e n}\right)$ \\
\cline { 2 - 7 } & $e_{\ell}$ & $\log _{2}\left(e_{\ell-1} / e_{\ell}\right)$ & $e_{\ell}$ & $\log _{2}\left(e_{\ell-1} / e_{\ell}\right)$ & $e_{\ell}$ & $\log _{2}\left(e_{\ell-1} / e_{\ell}\right)$ \\
\hline 6 & $3.5335 \mathrm{e}+01$ & $-1.9294 \mathrm{e}+00$ & $1.9512 \mathrm{e}-06$ & $3.0168 \mathrm{e}+00$ & $1.4095 \mathrm{e}-08$ & $3.9822 \mathrm{e}+00$ \\
7 & $1.3458 \mathrm{e}+02$ & $-1.9640 \mathrm{e}+00$ & $2.4107 \mathrm{e}-07$ & $3.0081 \mathrm{e}+00$ & $8.9187 \mathrm{e}-10$ & $3.9915 \mathrm{e}+00$ \\
8 & $5.2508 \mathrm{e}+02$ & $-1.9819 \mathrm{e}+00$ & $2.9965 \mathrm{e}-08$ & $3.0040 \mathrm{e}+00$ & $5.6070 \mathrm{e}-11$ & $3.9959 \mathrm{e}+00$ \\
9 & $2.0741 \mathrm{e}+03$ & $-1.9909 \mathrm{e}+00$ & $3.7354 \mathrm{e}-09$ & $3.0020 \mathrm{e}+00$ & $3.5144 \mathrm{e}-12$ & $3.9980 \mathrm{e}+00$ \\
10 & $8.2441 \mathrm{e}+03$ & $-1.9954 \mathrm{e}+00$ & $4.6629 \mathrm{e}-10$ & $3.0010 \mathrm{e}+00$ & $2.1996 \mathrm{e}-13$ & $3.9990 \mathrm{e}+00$ \\
11 & $3.2872 \mathrm{e}+04$ & $-1.9977 \mathrm{e}+00$ & $5.8247 \mathrm{e}-11$ & $3.0005 \mathrm{e}+00$ & $1.3757 \mathrm{e}-14$ & $3.9995 \mathrm{e}+00$ \\
12 & $1.3128 \mathrm{e}+05$ & $-1.9989 \mathrm{e}+00$ & $7.2784 \mathrm{e}-12$ & $3.0002 \mathrm{e}+00$ & $8.6012 \mathrm{e}-16$ & $3.9997 \mathrm{e}+00$ \\
13 & $5.2470 \mathrm{e}+05$ & $-1.9994 \mathrm{e}+00$ & $9.0965 \mathrm{e}-13$ & $3.0001 \mathrm{e}+00$ & $5.3767 \mathrm{e}-17$ & $3.9999 \mathrm{e}+00$ \\
\hline
\end{tabular}

Table 6: Smooth region, next to a jump: errors $e_{\ell}$ in (57) and estimates of the approximation order for $\hat{u}_{2 i+1}^{\ell+1}=R_{4}^{W E N O}\left(x_{i+\frac{1}{2}}^{\ell}, \varepsilon\right)$ with $\varepsilon_{\text {lin }}^{2}$ in (38) (left), $\varepsilon_{\text {alt }}^{2}$ in (39) (center) and $\varepsilon_{w e n}^{2}$ in (54) (right).

\begin{tabular}{|c|c|c|c|c|c|c|}
\hline \multirow{3}{*}{$\ell$} & \multicolumn{6}{|c|}{$\Omega_{0.5}=[0,0.5]$} \\
\cline { 2 - 7 } & $u_{i+\frac{1}{2}}-G_{4}^{W E N O}\left(x_{i+\frac{1}{2}}^{\ell}, \varepsilon_{l i n}\right)$ & $u_{i+\frac{1}{2}}-G_{4}^{W E N O}\left(x_{i+\frac{1}{2}}^{\ell}, \varepsilon_{a l t}\right)$ & $u_{i+\frac{1}{2}}-G_{4}^{W E N O}\left(x_{i+\frac{1}{2}}^{\ell}, \varepsilon_{w e n}\right)$ \\
\cline { 2 - 7 } & $e_{\ell}$ & $\log _{2}\left(e_{\ell-1} / e_{\ell}\right)$ & $e_{\ell}$ & $\log _{2}\left(e_{\ell-1} / e_{\ell}\right)$ & $e_{\ell}$ & $\log _{2}\left(e_{\ell-1} / e_{\ell}\right)$ \\
\hline 6 & $2.5632 \mathrm{e}-02$ & $6.3092 \mathrm{e}-02$ & $2.0519 \mathrm{e}-07$ & $2.8866 \mathrm{e}+00$ & $2.2037 \mathrm{e}-07$ & $2.9429 \mathrm{e}+00$ \\
7 & $2.4536 \mathrm{e}-02$ & $3.2641 \mathrm{e}-02$ & $2.7745 \mathrm{e}-08$ & $2.9472 \mathrm{e}+00$ & $2.8658 \mathrm{e}-08$ & $2.9717 \mathrm{e}+00$ \\
8 & $2.3987 \mathrm{e}-02$ & $1.6608 \mathrm{e}-02$ & $3.5973 \mathrm{e}-09$ & $2.9745 \mathrm{e}+00$ & $3.6532 \mathrm{e}-09$ & $2.9859 \mathrm{e}+00$ \\
9 & $2.3712 \mathrm{e}-02$ & $8.3781 \mathrm{e}-03$ & $4.5768 \mathrm{e}-10$ & $2.9875 \mathrm{e}+00$ & $4.6114 \mathrm{e}-10$ & $2.9930 \mathrm{e}+00$ \\
10 & $2.3575 \mathrm{e}-02$ & $4.2078 \mathrm{e}-03$ & $5.7710 \mathrm{e}-11$ & $2.9938 \mathrm{e}+00$ & $5.7925 \mathrm{e}-11$ & $2.9965 \mathrm{e}+00$ \\
11 & $2.3506 \mathrm{e}-02$ & $2.1086 \mathrm{e}-03$ & $7.2449 \mathrm{e}-12$ & $2.9969 \mathrm{e}+00$ & $7.2583 \mathrm{e}-12$ & $2.9982 \mathrm{e}+00$ \\
12 & $2.3472 \mathrm{e}-02$ & $1.0555 \mathrm{e}-03$ & $9.0755 \mathrm{e}-13$ & $2.9985 \mathrm{e}+00$ & $9.0839 \mathrm{e}-13$ & $2.9991 \mathrm{e}+00$ \\
13 & $2.3455 \mathrm{e}-02$ & $5.2803 \mathrm{e}-04$ & $1.1357 \mathrm{e}-13$ & $2.9992 \mathrm{e}+00$ & $1.1362 \mathrm{e}-13$ & $2.9996 \mathrm{e}+00$ \\
\hline
\end{tabular}

Table 7: Smooth region, next to a jump: errors $e_{\ell}$ in (57) and estimates of the approximation order for $\hat{u}_{2 i+1}^{\ell+1}=G_{4}^{W E N O}\left(x_{i+\frac{1}{2}}^{\ell}, \varepsilon\right)$ with $\varepsilon_{l i n}^{4}$ in (48) (left), $\varepsilon_{a l t}^{4}$ in (51) (center) and $\varepsilon_{w e n}^{4}$ in (53) (right).

6.3. A graphical investigation of the recursive application of the $M Q-R B F$ reconstruction techniques

It is interesting to carry out a recursive application of the interpolatory techniques considered in this Section for the function $u$ in (56). For this, we start from the resolution level $\ell=4$ (i.e., from the known 
values $\left\{u_{i}^{4}\right\}_{i=0}^{2^{4}}$ ), and apply recursively the interpolatory techniques considered in this Section in order to obtain a reconstructed function at the resolution level $\ell=8$ (i.e., $\left\{\hat{u}_{i}^{8}\right\}_{i=0}^{2^{8}}$ ). In Figure 1 , the circles represent the initial function values and the dotted lines are obtained with the recursive use of $\bar{R}_{2}^{i, 1}, G_{4}^{W E N O}$ and $R_{4}^{W E N O}$, for the different values of the shape parameter considered previously.

Note that linear techniques in the estimation of the shape parameter induce Gibbs oscillations next to the jump. The techniques with $O(1)$ approximation errors $\left(\bar{R}_{2}^{i, 1}, G_{4}^{W E N O}\right)$ induce $O(1)$ oscillations. The situation is much worse when using the $R_{4}^{W E N O}$ interpolatory technique with the linear estimation of the shape parameter. The observed $O\left(h^{-2}\right)$ error leads to a wild oscillatory behavior that invalidates this technique for practical use. The adaptive estimation of the shape parameter becomes mandatory in this case in order to obtain a sensible reconstruction. It is remarkable the good behavior observed when the (extremely cheap) $\varepsilon_{\text {alt }}$ option is implemented.
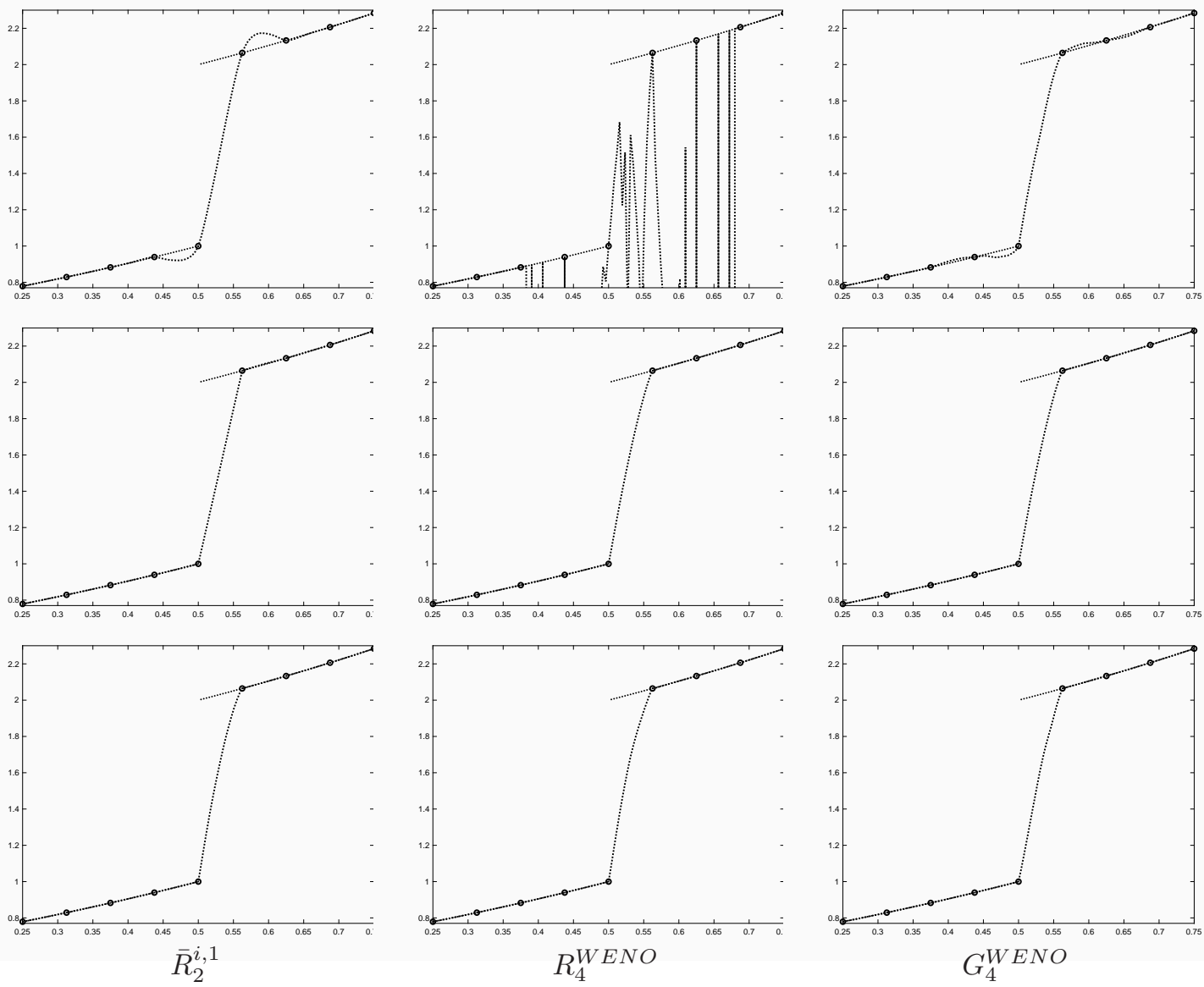

Figure 1: Central area of reconstruction (dotted lines) from 17 initial values of the test function in (56) obtained from the recursive use of $\bar{R}_{2}^{i, 1}, R_{4}^{W E N O}$ and $G_{4}^{W E N O}$ considering different approximations to $\varepsilon_{\text {opt }}$. First row: linear estimation of $\varepsilon_{\text {opt }}\left(\varepsilon_{\text {lin }}\right.$ in (32), (38), (48)); second row: $\varepsilon_{\text {alt }}$ from (33), (39), (51); third row: $\varepsilon_{w e n}$ from (36), (54), (53). 


\section{Conclusions and future work}

\subsection{Closing remarks}

We have carefully studied the theoretical accuracy of several MQ-RBF interpolatory techniques in the presence of discontinuities, showing that using linear estimates of the optimal shape parameter leads to a complete loss of accuracy in the interpolant next to jump discontinuities. Using ideas from polynomial WENO interpolation, we introduce adaptivity in the MQ-RBF interpolants and define, and study, several MQ-RBF-WENO techniques. We show that faithful approximations of discontinuous functions may be constructed using MQ-RBF-WENO interpolants, as long as the optimal shape parameter is computed in an adaptive way. Our MQ-RBF-WENO reconstruction techniques can accurately reconstruct piecewise smooth functions up to the interval containing a sufficiently isolated discontinuity, do not require to know or estimate in advance the discontinuity points and do not revert to WENO polynomial interpolation in the non smooth areas, as in $[12,13]$.

The numerical experiments confirm the theoretical results in the paper and suggest that this approach could be successfully used as a reconstruction technique in finite-difference schemes for conservation laws, as in $[12,13]$.

\subsection{Topics for future study}

The selection of WENO weights in this paper ensures an improved accuracy in the MQ-RBF WENO interpolant up to the interval containing a jump discontinuity. WENO weights that improve the accuracy of WENO interpolants around corner singularities have been proposed and analyzed in [2] and [1]. The use of these new weights could potentially lead to an improvement in the accuracy of an appropriately designed MQ-RBF WENO interpolant. However, due to the interplay between the error functions and the optimal shape parameters, further studies need to be carried out in order to incorporate these weights in our framework.

Other interesting topics for future work concern the inclusion of the proposed prediction step in finite difference numerical schemes for conservation laws and the generalization of our approach for the reconstruction of discontinuous multivariate functions from gridded data sets. The generalization is not straightforward since we need to study configurations of points that allow for an appropriate approximation of the optimal local shape parameters. Finally it is worthwhile to consider an extension of the strategy proposed here to scattered data which is the natural setting when using RBF based methods.

\section{Acknowledgement}

This research has been accomplished within Rete ITaliana di Approssimazione (RITA). The last two authors are members of the INdAM Research group GNCS, which has partially supported this work. The research of the two first authors was partially supported by Spanish MCINN MTM2017-83942-P.

We would like to thank the anonymous reviewers for their remarks and suggestions.

\section{References}

[1] S. Amat, J. Liandrat, J. Ruiz, and JC. Trillo, On a Power WENO Scheme with Improved Accuracy Near Discontinuities, SIAM J. Sci. Comput. 39-6 (2017), pp. A2472-A2507.

[2] S. Amat, J. Ruiz, New WENO smoothness indicators computationally efficient in the presence of corner discontinuities, J. Sci. Comput. 71 (2017), no. 3, 1265-1302.

[3] F. Aràndiga and A. M. Belda, Weighted ENO interpolation and applications, Communications in Nonlinear Science and Numerical Simulation 9 (2004) 187-195.

[4] F. Aràndiga, A. Cohen, R. Donat and N. Dyn, Interpolation and approximation of piecewise smooth functions, SIAM J. Numer. Anal. 43 (2005) 41-57.

[5] F. Aràndiga, A. M. Belda and P. Mulet, Point-value WENO multiresolution applications to stable image compression, J. Sci. Comput. 43(2) (2010) 158-182.

[6] M. Bozzini and M. Rossini, The detection and recovery of discontinuity curves from scattered data, J. Comput. Appl. Math. 240 (2013) 148-162. 
[7] M. Bozzini, L. Lenarduzzi and M. Rossini, Non-regular surface approximation. In: Floater M., Lyche T., Mazure ML., Mørken K., Schumaker L.L. (eds) Mathematical methods for curves and surfaces. Lecture Notes in Computer Science, vol. 8177, 68-87. Springer, Heidelberg, (2014).

[8] A. Crampton and J.C. Mason, Detecting and approximating fault lines from randomly scattered data, Numer. Algorithms 39 (13) (2005) 115-130.

[9] S. De Marchi, F. Marchetti and E. Perracchione, Jumping with variably scaled discontinuos kernels (VSDK), https://www.math.unipd.it/demarchi/papers/Draft_DMP_V8.2.pdf

[10] W. Erb, S. De Marchi, F. Marchetti, E. Perracchione and M. Rossini Shape-Driven Interpolation with Discontinuous Kernels: Error Analysis, Edge Extraction and Applications in MPI, (2019) arXiv:1903.03095 [math.NA].

[11] C. Gout, C. Le Guyader, L. Romani, A.-G. Saint-Guirons, Approximation of surfaces with fault(s) and/or rapidly varying data, using a segmentation process, Dm-splines and the finite element method, Numer. Algorithms 48 (13) (2008) 67-92.

[12] J. Guo and J.-H. Jung, A RBF-WENO finite volume method for hyperbolic conservation laws with the monotone polynomial interpolation method, Appl. Numer. Math., 112 (2017) 27-50.

[13] J. Guo, J.-H. Jung, Radial basis function ENO and WENO finite difference methods based on the optimization of shape parameters, J. Sci. Comput., 70 (2017) 551-575.

[14] A. Harten, B. Engquist, S. Osher, S. Chakravarthy, Some results on uniformly high-order accurate essentially nonoscillatory schemes, Applied Numerical Mathematics 2 (1986) 347-377.

[15] A. Harten, B. Engquist, S. Osher, S. Chakravarthy, Uniformly high order accurate essentially non-oscillatory schemes III, J. Comput. Phys. 71 (1987) 231-303.

[16] A. Harten, ENO schemes with subcell resolution, J. Comput. Phys. 83 (1989) 148-184.

[17] G.-S. Jiang and C-W Shu, Efficient implementation of weighted ENO schemes, J. Comput. Phys. 126 (1996) $202-228$.

[18] J.-H. Jung, A note on the Gibbs phenomenon with multiquadric radial basis functions, Appl. Numer. Math. 57 (2007), 213-219.

[19] E. Isaacson and H. B. Keller, Analysis of Numerical Methods. Wiley, New York, 1966

[20] L. Lenarduzzi and R. Schaback, Kernel-based adaptive approximation of functions with discontinuities, Appl. Math. Comput. 307 (2017) 113-123.

[21] X.-D. Liu, S. Osher and T. Chan, Weighted essentially non-oscillatory schemes, J. Comput. Phys. 115 (1994) $200-212$.

[22] L. Romani, M. Rossini, D. Schenone, Edge detection methods based on RBF interpolation, J. Comput. Appl. Math. 349 (2018), 532-547.

[23] C. W Shu, Essentially non-oscillatory and weighted essentially non-oscillatory schemes for hyperbolic conservation laws. In: Quarteroni, A. (ed.) Advanced Numerical Approximation of Nonlinear Hyperbolic Equations. Lecture Notes in Mathematics, vol. 1697, 325-432. Springer, Berlin, Heidelberg (1998) 